\title{
Forward-looking solvency contagion
}

\author{
Marco Bardoscia*a ${ }^{*}$ Paolo Barucca ${ }^{\mathrm{b}}$, Adam Brinley Codd ${ }^{\mathrm{a}}$, and John Hill ${ }^{\mathrm{c}}$

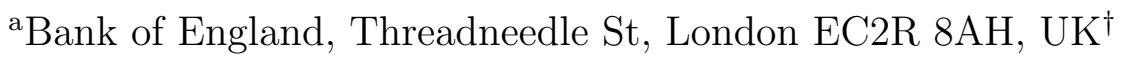 \\ ${ }^{\mathrm{b}}$ University College London, Gower St, London WC1E 6EA, UK \\ cSimudyne, 35 Luke St, London EC2A 4DS, UK
}

September 4, 2019

\begin{abstract}
Solvency contagion risk is a key channel through which systemic risk can come about. We introduce a model that accounts not only for losses transmitted after banks default, but also for losses due to the fact that creditors revalue their exposures when probabilities of default of their counterparties change. We apply the model to run a series of simplified stress tests of the UK banking system from 2008 to 2016, based on two datasets of real interbank exposures between the seven major UK banks. We show that risks due to solvency contagion decrease markedly from the peak of the crisis, to the point of becoming negligible. We also characterise the distributions of both vulnerabilities and systemic importances of individual banks, thereby tracking the evolution of risk concentration.
\end{abstract}

JEL classification: C63, G01, G12, G21, G28, G33

Keywords: Financial networks; Systemic risk; Financial contagion; Macro-prudential policy; Stress testing.

\footnotetext{
*marco.bardoscia@bankofengland.co.uk

${ }^{\dagger}$ Any views expressed are solely those of the author(s) and so cannot be taken to represent those of the Bank of England or to state Bank of England policy.
} 


\section{Introduction}

Financial institutions form interconnections in order to transfer and transform risk. But during a crisis, such links become channels through which distress can spread, turning an idiosyncratic shock into a systemic one. During the 2008 - 2009 financial crisis, one of the most potent of these mechanisms was solvency contagion. Financial institutions had lent large amounts of money to each other, both directly through interbank markets, and indirectly, through derivative markets. As institutions suffered losses, their creditworthiness deteriorated. Their counterparties reassessed the value of those claims and booked losses as a result. In this way, solvency shocks to one firm led quickly to solvency shocks at other firms.

This type of contagion does not require a firm to default in order to spread losses. Indeed, even at the peak of the crisis, there were very few occurrences of cascades of outright defaults. Instead, the fall in the creditworthiness of banks' counterparties was enough to lead to losses as banks revalued exposures that were marked-to-market. The Basel Commitee on Banking Supervision estimates that "roughly two-thirds of losses attributed to counterparty credit risk were due to CVA losses ${ }^{1}$ and only about one-third were due to actual defaults" (Basel Committee on Banking Supervision, 2011b).

Our contribution is twofold. First, we introduce a model for a forward-looking valuation of banks' equity in the presence of both ex-ante uncertainty on the value of non-interbank assets and information asymmetry about interbank assets, i.e. about the interconnectedness of other banks. The model provides a natural framework to describe pre-default solvency contagion in a setting in which banks can default not only at a fixed maturity, but at any point in time. This is consistent with post-crisis resolution frameworks, according to which banks can be put into resolution if they breach their minimum capital requirements. When a bank suffers an exogenous shock, its probability of default increases. The bank's counterparties then revalue its exposures to that bank. Because the valuation is forward-looking, it occurs even if the shock was not large enough to make the bank default, implying a reduction of the value in the interbank assets of its counterparties. Finally, the fall in value of interbank assets of counterparties is absorbed by their equity, resulting in pre-default contagion losses.

Second, we use our model to run a series of simplified stress tests, thereby showing that solvency contagion risks have declined sharply in the UK banking system over the period from 2008 to 2016. Scarcity of data on confidential interbank exposures means that empirical studies of financial contagion are relatively rare. In this paper, we benefit from access to two unique datasets of interbank exposures. This makes it, to the best of our knowledge, the only post-crisis empirical study of solvency contagion in the UK banking system. We also analyse two complementary aspects of how contagion losses

\footnotetext{
${ }^{1}$ Credit Valuation Adjustment (CVA) losses are precisely due to incorporating the risk that a counterparty can default into the mark-to-market value of assets.
} 
are distributed across banks: the vulnerability of individual banks and their systemic importance.

In most of the literature about stress testing and solvency contagion banks do not perform a forward-looking valuation. In this setting, banks do not try to estimate the probability of default of their counterparties, but they only observe whether they have defaulted or not. Hence, by definition, contagion can only happen post-defaults. The only form of uncertainty is about the liquidation value of interbank assets, and therefore on the recovery rate of those assets. Eisenberg and Noe (2001) provide the conceptual framework upon which most of the subsequent theoretical work is based. They provide a set of general conditions under which recovery rates can be uniquely determined endogenously. The model has been extended by Rogers and Veraart (2013) to the case in which there are explicit bankruptcy costs, by Suzuki (2002)) to the case in which banks have cross-holdings of equity, and by Fischer (2014) to the case in which liabilities have an arbitrary structure of seniorities. Elsinger et al. (2006a,b) are among the very few studies to explicitly account for the ex-ante uncertainty about the value of non-interbank assets. They perform a Monte Carlo sampling of the possible realisations of the financial system at maturity, to compute the solutions of the Eisenberg and Noe model for each realisation, and finally to average over all the sampled realisations. Our approach is based on the valuation framework introduced in Barucca et al. (2016) instead and it differs from Elsinger et al. (2006a,b) in two important aspects. First, the Monte Carlo sampling cannot be interpreted as a forward-looking valuation performed by banks, because it can be performed only by an entity with full knowledge of all interbank assets. In contrast, as in Barucca et al. (2016), we only require that banks have knowledge of their own interbank assets and that market prices of equities incorporate information about the updated valuations performed by all banks. Second, in Elsinger et al. (2006a,b), but also in Barucca et al. (2016), banks can only default at a fixed maturity, similarly to the Merton model (Merton, 1974). In our model, banks can default at any point in time, which corresponds to computing probabilities of default with the Black and Cox model (Black and Cox, 1976). As mentioned earlier, this is consistent with post-crisis resolution frameworks. In fact, regulators continuously monitor banks' capital and they can resolve, potentially at any point in time, a bank that breaches their minimum capital requirements. Earlier models of pre-default solvency contagion have been proposed in Battiston et al. (2012), Bardoscia et al. (2015, 2016, 2017), Fink et al. (2016). In those papers valuations of banks' equities are based on simple heuristic rules, while in our approach they are grounded in structural models of credit risk. For the sake of completeness we point out that pre-default contagion can occur in contexts unrelated to direct solvency contagion, such dynamic clearing (Banerjee et al., 2018) and fire sales (Cifuentes et al., 2005, Caccioli et al., 2014, Greenwood et al., 2015).

Empirical work on financial contagion has been hampered by the general lack of data about bilateral exposures between financial institutions. A partial solution is to adopt 
reconstruction techniques to infer exposures when only limited aggregate information about them is available. Such techniques have been pioneered in Upper and Worms (2004) and further developed in Hałaj and Kok (2013), Anand et al. (2015), Cimini et al. (2015), Gandy and Veraart (2016).

Most of the empirical papers using real interbank exposures are based on an even simpler contagion mechanism than the one introduced in Eisenberg and Noe (2001). Distress is propagated only through defaults, and the recovery rate is exogenous and in most cases it is set to zero. This means that creditors lose the full face value of their claims towards defaulted banks. That mechanism has been applied to several banking systems: US (Furfine, 2003), Germany (Upper and Worms, 2004), UK (Wells, 2004), Belgium (Degryse et al., 2007), Brazil (Cont et al., 2010), Italy (Mistrulli, 2011), EU (Hüser et al., 2018). ${ }^{2}$ Notable exceptions are the aforementioned Elsinger et al. (2006a,b) that extend the Eisenberg and Noe model by incorporating the ex-ante uncertainty about non-interbank assets to study the Austrian and to the UK banking system. ${ }^{3}$ De Souza et al. (2016) adopt a mixed approach that consists in triggering contagion by assuming selective defaults and in weighting contagion losses with the probabilities that such triggering defaults occur. While probabilities of triggering defaults are estimated with a mix of strategies (including the Merton model), contagion losses are computed using DebtRank (Battiston et al., 2012). Both the previous empirical studies of the UK banking system use pre-crisis data. Wells (2004) finds that, while idiosyncratic shocks rarely cause cascades of defaults, they can be responsible for material losses to a significant fraction of banks. Elsinger et al. (2006b) show that idiosyncratic shocks can have a much lower impact than systematic shocks.

In the literature on systemic risk there is no widely accepted methodology to characterise the systemic importance of an institution. Approaches based on loss distributions, such as those proposed in Kurth and Tasche (2003), Glasserman (2005), Adrian and Brunnermeier (2016), Acharya et al. (2013) are not feasible in our case, as our model produces point estimates. Instead we perform a series of simple experiments in which each bank is selectively removed from the banking system to assess its marginal contribution to contagion losses. We check the robustness of our results also by performing the Shapley decomposition of contagion losses (Tarashev et al., 2010).

We refer the reader interested in a more comprehensive account of the literature on financial contagion to Glasserman and Young (2016), which provides an excellent general review, and to Upper (2011), which focuses on summarising empirical results. Here we only mention that, despite the abundance of works investigating the role that the topology of the underlying network of contracts has on the propagation of distress, there is little consensus, for example, as to whether more interconnected networks are

\footnotetext{
${ }^{2}$ Upper and Worms (2004) fully reconstruct bilateral exposures, while Degryse et al. (2007) and Wells (2004) use reconstruction techniques to supplement partial data about actual bilateral exposures.

${ }^{3}$ In both cases bilateral exposures are partially reconstructed.
} 
more resilient or not (Allen and Gale, 2000, Freixas et al., 2000, Cifuentes et al., 2005). More recent studies find that interconnectedness might have a non-monotonic impact on resilience (Nier et al., 2007, Gai and Kapadia, 2010, Elliott et al., 2014) or point towards an even more nuanced behaviour (Amini et al., 2013, Acemoglu et al., 2015, Bardoscia et al., 2017). Glasserman and Young (2015) follow a different approach that allows to give bounds on losses due to solvency contagion by using only information about single institutions.

The paper is organised as follows: in section 2 we introduce the modelling framework based on valuation functions, in section 3 we describe the data used and explain how to estimate the model parameters, in section 4 we present our results, while in section 5 we draw some conclusions and outline possible policy implications of our work.

\section{Model}

Our approach follows the general framework introduced in Barucca et al. (2016). We will depart significantly from it in section 2.2 , specifically when we introduce a different dynamic for bank defaults. Our financial system consists of $n$ banks, each represented with a stylised balance sheet. The asset side of the balance sheet of bank $i$ is divided into external assets $A_{i}^{e}$ (e.g. loans to households and corporates) and interbank assets (e.g. claims on other banks, such as interbank loans). Similarly, the liability side is comprised of external liabilities $L_{i}^{e}$ (e.g. household and corporate deposits), interbank liabilities (e.g. money borrowed on interbank markets), and equity $E_{i}$. Interbank assets and liabilities represent obligations between banks. Interbank assets are represented by the matrix $A$, whose element $A_{i j}$ is the value of the obligation of bank $j$ to bank $i$. The total interbank assets of bank $i$ are simply $\sum_{j=1}^{n} A_{i j}$. Symmetrically, interbank liabilities are represented by the matrix $L$ whose element $L_{i j}$ is the value of the obligation of bank $i$ to bank $j$. The total interbank liabilities of bank $i$ are $\sum_{j=1}^{n} L_{i j}$. Given that any interbank asset corresponds to an interbank liability (and vice versa), we have that $A_{i j}=L_{j i}$, for all $i$ and $j$. The diagonal elements of both matrices are equal to zero, i.e. $A_{i i}=L_{i i}=0$, for all $i$, simply meaning that a bank cannot have an obligation to itself.

The matrix of interbank assets $A$ can be interpreted as the weighted adjacency matrix of a network whose nodes represent $n$ banks and whose edges represent bilateral exposures between them. This approach is common to the vast majority of papers modelling financial contagion, see e.g. Glasserman and Young (2016) for a comprehensive review.

We assume that each bank does not know the composition of interbank assets and liabilities of other banks. Aggregate quantities, such the external assets and liabilities and total interbank assets and liabilities of all banks, are known to all banks instead. ${ }^{4}$ Such information asymmetry means that each bank is unable to work out in isolation the

\footnotetext{
${ }^{4}$ Indeed such aggregate quantities can be taken from financial statements, see section 3 .
} 
value of its interbank assets. This is because the value of its interbank assets depends on the solvency of its own counterparties. In turn, the solvency of those counterparties depends on the value of their interbank assets, which depends on the solvency of their own counterparties, and so on. In this way solvencies of all banks depend circularly on the solvencies of all banks. Because each bank does not have any knowledge of what the counterparties of other banks are, it is not able to determine by itself whether any other bank is solvent, including its counterparties. It is worth remarking that this lack of capability to determine autonomously the value of interbank assets does not depend on the existence of an ex-ante (stochastic) uncertainty about the intrinsic value of interbank assets, but rather on banks' lack of complete knowledge about the underlying network of obligations, i.e. about the interconnectedness of other banks. Moreover, the model accounts for the ex-ante uncertainty about the value of external assets due to fact that at time $t$ banks perform a forward-looking valuation of their equities over an horizon $T-t$, with $t<T$.

Each bank performs a risk-neutral valuation of its interbank assets, which is in turn based on its risk-neutral valuation of the probability of default of its counterparties. Because of the limited knowledge that each bank has about the network of obligations, its initial valuation will account only for the probabilities of default of its direct counterparties. Banks subsequently perform additional valuation rounds that allow them to incorporate into their valuations, albeit only indirectly, probabilities of default of banks that are not their direct counterparties.

We build the model in two steps. First, in section 2.1 we illustrate how banks perform the valuation of their interbank assets collectively in the absence of any ex-ante uncertainty on the value of external assets. Second, in section 2.2 we introduce the uncertainty about the value of external assets.

\subsection{Valuation of counterparty risk}

At a given time $T$, the balance sheet identity for bank $i$ reads:

$$
A_{i}^{e}(T)+\sum_{j=1}^{n} A_{i j}(T)=L_{i}^{e}(T)+\sum_{j=1}^{n} L_{i j}(T)+E_{i}(T) .
$$

A key observation is that in (1) interbank assets and liabilities have been taken at face value. In other words, $E_{i}(T)$ is the equity implied by the assumption that all bank $i$ 's counterparties will make good on their obligations and that bank $i$ will make good on its obligations towards its own counterparties.

We assume that bank $i$ evaluates how much its interbank assets and liabilities would be worth if its counterparties defaulted. Let us denote such valuations with $\tilde{A}_{i j}(T)$ and $\tilde{L}_{i j}(T)$ respectively. By plugging those into (1), bank $i$ works out $\tilde{E}_{i}(T)$, the value of 
its equity implied by those valuations:

$$
\tilde{E}_{i}(T)=A_{i}^{e}(T)+\sum_{j=1}^{n} \tilde{A}_{i j}(T)-L_{i}^{e}(T)-\sum_{j=1}^{n} \tilde{L}_{i j}(T) .
$$

We assume that interbank liabilities are always taken at face value, i.e. that $\tilde{L}_{i j}(T)=$ $L_{i j}(T)$. In other words, when banks recognize that their counterparties might not be solvent they are not allowed to boost the valuations of their own equities by discounting the corresponding interbank liabilities. This amounts to assuming that, when a bank defaults, receivers will still try to recover the full (face) value of all obligations towards that banks. Therefore, debtors cannot expect to be able to write off some of their interbank liabilities towards the defaulted bank.

As regards interbank assets, we assume that valuations are made in the following way:

$$
\begin{gathered}
\tilde{A}_{i j}(T)=A_{i j}(T) \mathbb{V}_{j}\left(\tilde{E}_{j}(T)\right) \\
\mathbb{V}_{j}\left(\tilde{E}_{j}(T)\right)= \begin{cases}1 & \text { for } \tilde{E}_{j}(T) \geq 0 \\
\rho_{j}\left(\tilde{E}_{j}(T)\right) & \text { for } \tilde{E}_{j}(T)<0\end{cases}
\end{gathered}
$$

with $\rho_{j}(x)<1$, for all $x$. The functions $\mathbb{V}_{j}$ are called valuation functions. In other words, if bank $j$ is expected to be solvent, i.e. if the valuation of its equity is larger than zero, then the interbank asset $A_{i j}(T)$ is worth its face value. Otherwise, the interbank asset $A_{i j}(T)$ is worth less than its face value, where $\rho_{j}$ is the recovery function of bank $j$ 's obligations, i.e. one minus its loss given default.

An important observation is that the valuation of bank $i$ 's interbank asset towards $j$ depends on the valuation of bank $j$ 's equity. In fact, by doing so, bank $i$ is incorporating the valuation of bank $j$ 's interbank assets into the valuation of its own equity. If bank $i$ used the value of bank $j$ 's equity implied by (1), it would be ignoring the possibility that bank $j$ 's counterparties could not make good on their own obligations towards $j$.

As both sides of the system of equations (2) (one equation for each bank) depend on the vector $\tilde{\mathbf{E}}(T),(2)$ is a set of fixed point equations for $\tilde{\mathbf{E}}(T)$ and solutions of (2) can be thought of as equilibria for valuations of equities. This means that in a financial system in which valuations of interbank assets depend on valuations of counterparties' equities, everybody's (valuation of) equities depends self-consistently on everybody's (valuation of) equities.

In Barucca et al. (2016) it is shown that, by suitably choosing the recovery functions, (2) is equivalent to contagion models previously proposed in the literature. For example, by choosing exogenous recovery functions $\rho_{j}\left(E_{j}(T)\right)=\rho_{j}$ one recovers the model in Furfine (2003), Cont et al. (2013). Instead, by choosing the endogenous recovery functions $\rho_{j}\left(E_{j}(T)\right)=\left(1+\frac{E_{j}(T)}{L_{j}^{e}(T)+\sum_{k} L_{j k}(T)}\right)^{+}$one recovers the model in Eisenberg and Noe (2001). 
While the solution of (2) is not necessarily unique, if valuation functions satisfy certain conditions, there exists the greatest solution. The greatest solution is simultaneously optimal for all banks, in the sense that all banks attain the equity whose value is maximal across all the solutions. In particular, in Barucca et al. (2016) it is shown that if valuation functions are feasible, i.e. if they (i) take values in the interval $[0,1]$, (ii) are non-decreasing functions of the valuation of equities, and (iii) are continuous from above, then (2) admits the greatest solution. Moreover, in this case the greatest solution can be easily computed iteratively by choosing as starting point $\tilde{\mathbf{E}}^{(0)}(T)=\mathbf{E}(T)$, i.e. the vector of equities implied by (1) (where all interbank assets are taken at their face value) and by repeatedly applying (2) to compute the subsequent iterations $\tilde{\mathbf{E}}^{(1)}(T), \tilde{\mathbf{E}}^{(2)}(T), \ldots$, until convergence is reached.

Such iterative procedure can be interpreted as banks collectively working out the greatest solution. In practice, each bank would start from the baseline assumption that its interbank assets are worth their face value, i.e. $\tilde{\mathbf{E}}^{(0)}(T)=\mathbf{E}(T)$. Eq. (3) would then be used to compute an updated estimate for the valuation of interbank assets which, when plugged into (2), would yield an updated estimate for the valuation of equities $\tilde{\mathbf{E}}^{(1)}(T)$. Banks would then iterate those steps, every time plugging $\tilde{\mathbf{E}}^{(k-1)}(T)$ into (3) to obtain a new estimate for the valuation of its interbank assets and using (2) to get a new estimate $\tilde{\mathbf{E}}^{(k)}(T)$ of their valuation of equities, until convergence. It is worth remarking that, in order to compute updated estimates for the valuation of interbank assets and equities, banks do not need to learn the updated estimates of their own counterparties' equities, but only whether those updated estimates are positive or not. This would happen if, for example, whenever a bank learned that the valuation of equity worth were negative, it would make this piece of information public, or at least it would inform its counterparties.

Valuation functions (3b) are clearly feasible as long as recovery functions are nondecreasing and continuous from above. This is true, for example, for both the aforementioned Furfine and Eisenberg and Noe valuation functions.

We now observe that the greatest solution for valuation functions of the form (3b) can be easily computed when $\mathbf{E}(T) \geq 0$, i.e. when equities taken at face value are all

larger than zero. In fact, since $\tilde{E}_{j}^{(0)}(T) \geq 0$, for all $j$, from $(3 \mathrm{~b})$ we get $\mathbb{V}_{j}\left(\tilde{E}_{j}^{(0)}(T)\right)=1$, for all $j$. Hence, $\tilde{\mathbf{E}}^{(1)}(T)=\mathbf{E}(T)=\tilde{\mathbf{E}}^{(0)}(T)$, meaning that convergence is reached and that the greatest solution is $\mathbf{E}(T)$. As a consequence, for valuation functions of the form (3b), the greatest solution can be different from the face value of equities only if there is at least one bank whose equity, when taken at face value, is smaller than zero.

\subsection{Ex-ante valuation}

So far, we have assumed external assets and liabilities to be known with certainty. However, if $T$ is in the future and if we want to perform a forward looking valuation 
at any time $t<T$, there will be some ex-ante uncertainty about the value of external assets and liabilities. To model the ex-ante uncertainty about external assets we assume that they follow geometric Brownian motions. Here we will discuss the case in which external assets are independent, i.e. $\mathrm{d} A_{i}^{e}(s)=\mu_{i} A_{i}^{e}(s) \mathrm{d} s+\sigma_{i} A_{i}^{e}(s) \mathrm{d} W_{i}(s), \forall i$ and for $s \in[t, T]$ and in which other assets do not bear any interest. A more general case will be discussed in Appendix A. We take external liabilities to be non-stochastic, but we point out that we do so without any loss of generality. ${ }^{5}$

Given that $\mathbf{A}^{e}(T)$ is a vector of random variables, from (2) also $\tilde{\mathbf{E}}(T)$ is a vector of random variables. We assume that no arbitrage opportunities exist, that the market is complete, and that banks behave as risk-neutral investors. Therefore, they perform the valuation of equities at $t<T$ by computing their expectation under the (unique) Equivalent Martingale Measure $\mathbb{Q}$, conditional on the information available at time $t$, i.e. conditional on the realisation at time $t$ of the stochastic process followed by external assets: $\tilde{\mathbf{E}}(t) \equiv \mathbb{E}^{\mathbb{Q}}\left[\tilde{\mathbf{E}}(T) \mid \mathbf{A}^{e}(t)\right] \cdot 6,7$

We now proceed to compute the conditional expectation of all terms in (2). External assets are a martingale under the measure $\mathbb{Q}$ and $\mathbb{E}^{\mathbb{Q}}\left[A_{i}^{e}(T) \mid \mathbf{A}^{e}(t)\right]=A_{i}^{e}(t)$. The only stochastic dependence in interbank assets $\tilde{A}_{i j}(T)$ is in the valuation function. Additionally, since interbank assets do not bear any interest, $A_{i j}(t)=A_{i j}(T), \forall i, j$. All liability terms do not have any stochastic dependence and do not bear interest, hence: $L_{j}^{e}(t)=L_{j}^{e}(T)$, and $L_{i j}(t)=L_{i j}(T), \forall i, j$. By putting all together we have:

$$
\tilde{E}_{i}(t)=A_{i}^{e}(t)+\sum_{j=1}^{n} A_{i j}(t) \mathbb{E}^{\mathbb{Q}}\left[\mathbb{V}_{j}\left(\tilde{E}_{j}(T)\right) \mid \mathbf{A}^{e}(t)\right]-L_{i}^{e}(t)-\sum_{j=1}^{n} L_{i j}(t),
$$

where we are left with the task to compute the conditional expectation of valuation functions.

In Barucca et al. (2016) the conditional expectations of valuation functions in (4) are computed under the assumption that the valuation functions in (3b) are those corresponding to the Eisenberg and Noe model, which are characterised by endogenous recovery functions. As pointed out in Cont et al. (2013), models with such recovery functions assume that banks' assets are simultaneously liquidated without incurring in any additional bankruptcy costs. This can lead to overestimating realised recovery rates and therefore to overestimating equity valuations. Our approach is to use exogenous recovery functions instead and to explore a range of values for the recovery rates $\rho_{j}$.

\footnotetext{
${ }^{5}$ In fact, one could easily consider the difference between external assets and external liabilities as a single stochastic process and put the external liabilities equal to zero. This is the approach taken e.g. in Eisenberg and Noe (2001), in which the cash flow can be interpreted as the difference between external assets and external liabilities.

${ }^{6}$ In order to keep the notation light, we do not explicitly distinguish between random variables and their values. For example, $\mathbb{E}^{\mathbb{Q}}\left[A_{j}^{e}(T) \mid A_{j}^{e}(t)\right]$ is the expectation of the random variable $A_{j}^{e}(T)$ conditional on the realisation of the random variable $A_{j}^{e}(t)$.

${ }^{7}$ The assumption that non-stochastic assets and liabilities do not bear any interest is consistent with a zero risk-free rate. For this reason no discounting factor appears in the conditional expectation.
} 
In particular, this allows us to investigate the worst-case scenario by setting all recovery rates equal to zero. If recovery functions in (3b) are exogenous, the conditional expectations of valuation functions in (4) read:

$$
\begin{aligned}
\mathbb{E}^{\mathbb{Q}}\left[\mathbb{V}_{j}\left(E_{j}(T)\right) \mid \mathbf{A}^{e}(t)\right] & =\mathbb{E}^{\mathbb{Q}}\left[\mathbb{1}_{\tilde{E}_{j}(T) \geq 0}+\rho_{j} \mathbb{1}_{\tilde{E}_{j}(T)<0} \mid \mathbf{A}^{e}(t)\right] \\
& =\mathbb{E}^{\mathbb{Q}}\left[\mathbb{1}_{\tilde{E}_{j}(T) \geq 0} \mid \mathbf{A}^{e}(t)\right]+\rho_{j} \mathbb{E}^{\mathbb{Q}}\left[\mathbb{1}_{\tilde{E}_{j}(T)<0} \mid \mathbf{A}^{e}(t)\right] \\
& =\mathbb{E}^{\mathbb{Q}}\left[\mathbb{1}_{\tilde{E}_{j}(T) \geq 0} \mid \mathbf{A}^{e}(t)\right]+\rho_{j} \mathbb{E}^{\mathbb{Q}}\left[\left(1-\mathbb{1}_{\tilde{E}_{j}(T) \geq 0}\right) \mid \mathbf{A}^{e}(t)\right] \\
& =\rho_{j}+\left(1-\rho_{j}\right) \mathbb{E}^{\mathbb{Q}}\left[\mathbb{1}_{\tilde{E}_{j}(T) \geq 0} \mid \mathbf{A}^{e}(t)\right],
\end{aligned}
$$

where the only conditional expectation left to compute is, by definition, the probability that the valuation of bank $j$ 's equity is positive at $T$, conditional on the value of external assets at time $t$ (see Appendix A for the explicit expression). The second line of (5) means that the valuation of the interbank assets is the sum of two contributions, the face value of the asset weighted by bank $j$ 's survival probability, and the recovery rate weighted by bank $j$ 's probability of default.

In general, because valuation functions in (3b) depend only on $\tilde{\mathbf{E}}(T)$, i.e. on the valuations of equities at $T$, their conditional expectations in (2) depend only the conditional distributions of $\tilde{\mathbf{E}}(T)$, and not on their entire stochastic trajectories $\{\tilde{\mathbf{E}}(s)\}_{t \leq s \leq T}$. Indeed, all trajectories such that $\tilde{E}_{j}(T) \geq 0$ contribute equally to the conditional expectation in (3b), regardless on whether $\tilde{E}_{j}(s)$ has become smaller than zero for $s$ between $t$ and $T$. In other words, if the equity valuation of a bank becomes smaller than zero before $T$, that bank can "gamble for resurrection", hoping that the stochastic fluctuations of its external assets bring its equity valuation above zero at $T$.

However, in practice the post-crisis resolution framework(s) (Bank of England, 2014) implies that banks' equity levels are constantly monitored by regulators and, if they fall below a certain buffer, banks can be put into resolution. Moreover, since banks are reliant on their ability to roll-over their liabilities, banks with little equity would run into problems in funding markets before they were truly insolvent. This was most clearly demonstrated during the crisis by the failure of Northern Rock, and explained in Shin (2009). As a consequence, in reality defaults can occur whenever equities become equal to zero, which can happen at any point in time, including before $T$. Therefore, a more realistic definition of default of bank $j$ should depend not only on the valuation of its equity at $T$, but on whether this has become negative at any time before $T$. We define $\tau_{j}$, the default time of bank $j$ as:

$$
\tau_{j}=\inf \left\{s \in[t, T]: \tilde{E}_{j}(s)<0\right\}
$$

i.e. as the earliest time at which the valuation of its equity becomes negative.

We now want to capture the situation in which, if the valuation of bank $j$ 's equity is 
positive until $T$, i.e. if $\tau_{j}>T$, then bank $j$ does not default and its interbank assets are taken at face value. Conversely, if at any point in time before $T$ (or at $T$ ) bank $j$ 's the valuation of bank $j$ 's equity is smaller than zero, i.e. if $\tau_{j} \leq T$, then bank $j$ defaults and its interbank assets are discounted by the recovery rate. By using the same formalism in Bielecki et al. (2004), Brigo and Tarenghi (2004), this corresponds to replacing the conditional expectations in (4) with:

$$
\begin{aligned}
\mathbb{E}^{\mathbb{Q}}\left[\mathbb{1}_{\tau_{j}>T}+\rho_{j} \mathbb{1}_{\tau_{j} \leq T} \mid \mathbf{A}^{e}(t)\right] & =\mathbb{E}^{\mathbb{Q}}\left[\mathbb{1}_{\tau_{j}>T}+\rho_{j}\left(1-\mathbb{1}_{\tau_{j}>T}\right) \mid \mathbf{A}^{e}(t)\right] \\
& =\rho_{j}+\left(1-\rho_{j}\right) \mathbb{E}^{\mathbb{Q}}\left[\mathbb{1}_{\tau_{j}>T} \mid \mathbf{A}^{e}(t)\right]
\end{aligned}
$$

It is worth noting that in both (5) and (7), if $\rho_{j}$ were equal to one, the conditional average of the valuation function would be identically equal to one, meaning that claims towards bank $j$ would never be re-evaluated, because its counterparties would always expect to recover the full face value of their claims, even when bank $j$ would default. This would correspond to the situation in which all the proceeds from the liquidation of bank $j$ 's assets would be enough to pay back all its liabilities, effectively implying that the liquidation process would not entail any additional bankruptcy costs. Ultimately in our model the revaluation of interbank assets is triggered by the belief that the liquidation process will entail non-zero bankruptcy costs, i.e. that recovery rates will be smaller than one.

The problem of computing the survival probability in (7) has been solved first in the context of credit structural models in Black and Cox (1976). In order to use the result in Black and Cox (1976) we need to recast the condition $\tilde{E}_{j}(s)$ in terms of quantities known at time $t$. The starting point is (4) at time $s$ : $\tilde{E}_{j}(s)=$ $A_{j}^{e}(s)+\sum_{k=1}^{n} \tilde{A}_{j k}(s)-L_{j}^{e}(s)-\sum_{k=1}^{n} L_{j k}(s)$. Now, let us imagine that bank $i$ is computing bank $j$ 's survival probability in order to evaluate its interbank asset $A_{i j}$. In general, bank $i$ has no knowledge of bank $j$ 's own interbank assets, and therefore it is not able to compute the term $\sum_{k=1}^{n} \tilde{A}_{j k}(s)$ directly. Here we assume that bank $i$ is not attempting to perform a direct valuation of bank $j$ 's interbank assets, but only of its own interbank assets. In other words, the valuation of bank $j$ 's interbank assets enters into the valuation of bank $i$ 's interbank assets only indirectly, through the valuation of bank $j$ 's equity. This corresponds to assuming that, when evaluating bank $j$ 's valuation function, bank $i$ performs the following approximation: $\sum_{k=1}^{n} \tilde{A}_{j k}(s) \approx \sum_{k=1}^{n} \tilde{A}_{j k}(t)=\tilde{E}_{j}(t)-A_{j}^{e}(t)+L_{j}^{e}(t)+\sum_{k=1}^{n} L_{j k}(t)$. However, bank $j$ does not perform such an approximation and indeed it performs a valuation of its interbank assets by computing the valuation function of its own counterparties. By using the approximation we get: $\tilde{E}_{j}(s) \approx \tilde{E}_{j}(t)+A_{j}^{e}(s)-A_{j}^{e}(t)$, and therefore the survival condition reads: $A_{j}^{e}(s) \geq A_{j}^{e}(t)-\tilde{E}_{j}(t), \forall s \in[t, T]$. We can now adapt the result in 
Black and Cox (1976) (see Appendix A):

$$
\mathbb{E}^{\mathbb{Q}}\left[\mathbb{1}_{\tau_{j}>T} \mid \mathbf{A}^{e}(t)\right]= \begin{cases}0 & \text { for } \tilde{E}_{j}(t) \leq 0 \\ \mathcal{N}\left[\frac{\left.\log \frac{A_{j}^{e}(t)}{A_{j}^{e}(t)-\tilde{E}_{j}(t)}-\frac{\sigma_{j}^{2}(T-t)}{2}\right]}{\sigma_{j} \sqrt{(T-t)}}\right] & \\ -\frac{A_{j}^{e}(t)}{A_{j}^{e}(t)-E_{j}(t)} \mathcal{N}\left[\frac{\left.\log \frac{A_{j}^{e}(t)-\tilde{E}_{j}(t)}{A_{j}^{e}(t)}-\frac{\sigma_{j}^{2}(T-t)}{2}\right]}{\sigma_{j} \sqrt{(T-t)}}\right] & \\ 1 & \text { for } 0<\tilde{E}_{j}(t)<A_{j}^{e}(t), \\ & \text { for } \tilde{E}_{j}(t) \geq A_{j}^{e}(t)\end{cases}
$$

where $\mathcal{N}$ is the cumulative distribution of a Gaussian random variable with mean equal to zero and variance equal to one.

From (8) we can see that the expectation on the right-hand side of (7) is a function of $\tilde{E}_{j}(t)$, the equity valuation of bank $j$ at time $t$. Indeed, one can show that, as a function of $\tilde{E}_{j}(t)$, such expectation is itself a feasible valuation function (see Appendix $\mathrm{B}$, where we also prove that the expectation on the right-hand side of (7) is a feasible valuation function). As a result, (4) also admits a greatest solution, which analogously to the greatest solution of (2) is computed iteratively by choosing the equities implied by taking interbank assets at their face value, i.e. $\tilde{\mathbf{E}}^{(0)}(t)=\mathbf{E}(t) \equiv$ $A_{i}^{e}(t)+\sum_{j=1}^{n} A_{i j}(t)-L_{i}^{e}(t)+\sum_{j=1}^{n} L_{i j}(t)$. Also in this case it is possible to interpret the iterative procedure as banks collectively working out the greatest solution, but with an important difference. Indeed here, in order to compute updated estimates for the valuation of interbank assets and equities, banks need to promptly learn the updated estimates of the equities of their counterparties, not only whether those are positive or not. This would happen for example if markets were efficient, in the sense that, as banks would update their estimates of valuation of equities, prices of equity and debt would incorporate the information about the creditworthiness of counterparties. ${ }^{8}$

We refer to the model with valuation functions equal to the conditional expectations in (7) as to the exogenous Black and Cox network valuation model, because it features exogenous recovery functions and the default dynamics of the Black and Cox model, i.e. banks can default at any point in time. While most results in section 4 are based on this model, we will make comparisons with two additional models of ex-ante network valuation. First, the model in Barucca et al. (2016), which we refer to as the endogenous

\footnotetext{
${ }^{8}$ Strictly speaking, the market price of the equity should reflect the fact that equity holders have limited liability, meaning that the equity cannot become negative However, in our model equities can be negative and indeed they should be thought of as the value of the net worth (assets minus liabilities), rather than as the payoff of shareholders. Nevertheless, the implied market value of such net worths can be inferred by observing market prices of both equity and debt. Indeed, at maturity, by denoting with $A$ and $L$ the face values of assets and liabilities, the value of equity is $\max (A-L, 0)$, while the value of debt is $\min (L, A)$. Given that the net worth is defined as $N=A-L$, one has that $N=\max (A-L, 0)+\min (L, A)-L$. Before maturity, the identity still holds by replacing all the terms with their conditional expectations.
} 
Merton network valuation model, because it features endogenous recovery functions (à la Eisenberg and Noe) and the default dynamics of the Merton model, i.e. banks can default only at $T$. Second, the model with valuation functions equal to the conditional expectations in (5), which we refer to as the exogenous Merton network valuation model, because it features exogenous recovery functions and the default dynamics of the Merton model.

\subsection{Shocks}

In section 4 we will apply the model by carrying out simplified stress test exercises. The basic idea is to assume again that an exogenous shock hits external assets:

$$
\mathbf{A}^{e}(t) \rightarrow \mathbf{A}^{e}(t)+\Delta \mathbf{A}^{e},
$$

where $\Delta A_{i}^{e}<0$, for all $i$. Shocks to external assets will be absorbed by equity in the first instance generating the equity loss $\Delta \mathbf{E}^{\text {shock }}=\Delta \mathbf{A}^{e} \cdot{ }^{9}$ In order to compute the additional losses due to the revaluation of interbank assets one simply has to compute the fixed point $\tilde{\mathbf{E}}^{*}(t)$ of (4) using the post-shock vector of equities as starting point. The total loss will therefore include two contributions, the exogenous shock $\Delta \mathbf{E}^{\text {shock }}$ and the losses due to the contagion mechanism, i.e. $\Delta \mathbf{E}^{\text {cont }}=\tilde{\mathbf{E}}^{(0)}(t)-\mathbf{E}^{*}(t)$.

An important observation is that, for valuation functions of the form (3b), i.e. in absence of ex-ante uncertainty (and therefore of a forward-looking valuation), if postshock equities are all positive, then $\tilde{\mathbf{E}}^{(0)}(t)=\mathbf{E}^{*}(t)$ (see end of section 2.1). This means that, if shocks to external assets are less than the equity taken at face value for all banks, losses due to the contagion will be equal to zero. In other words, losses due to the contagion can be larger than zero only if the exogenous shock is so large that it is sufficient alone to put at least one bank in default. As shown by our results in section 4 , this does not apply to valuation functions discussed in section 2.2, which can imply losses due to the contagion also for smaller exogenous shocks.

Interestingly, the losses due to contagion can be further decomposed by means of the following observation. $\tilde{\mathbf{E}}^{(1)}(t)$, i.e. the vector of valuations of equities computed after iterating (4) once, incorporates the valuations performed by each bank of its own direct counterparties. $\tilde{\mathbf{E}}^{(2)}(t)$, the vector of valuations of equities computed after iterating (4) twice, captures subsequent revaluations performed by each bank's counterparties of their own counterparties. As a consequence, by measuring $\tilde{\mathbf{E}}^{(1)}(t)$ one can isolate the effects of direct losses, i.e. the losses caused by direct counterparties, from the losses genuinely caused by additional rounds of contagion propagation, i.e. by the amplification due to the network contribution.

\footnotetext{
${ }^{9}$ Even though in principle the shock in external assets could be larger than the equity, we always have $\left|\Delta A_{j}^{e}\right|<E_{j}(t)$. In fact, since our main purpose is to study contagion mechanisms, we are not interested in the case in which the exogenous shock alone is sufficient to make banks default.
} 
In summary, by denoting the vector of equities before the exogenous shock hits with $\mathbf{E}^{\text {pre-shock }}(t)$, there are three different contributions to the total losses:

$$
\begin{aligned}
& \Delta \mathbf{E}^{\text {shock }}=\mathbf{E}^{\text {pre-shock }}(t)-\tilde{\mathbf{E}}^{(0)}(t) \\
& \Delta \mathbf{E}^{\text {direct }}=\tilde{\mathbf{E}}^{(0)}(t)-\tilde{\mathbf{E}}^{(1)}(t) \\
& \Delta \mathbf{E}^{\mathrm{amp}}=\tilde{\mathbf{E}}^{(1)}(t)-\tilde{\mathbf{E}}^{*}(t),
\end{aligned}
$$

while the losses due to contagion are simply $\Delta \mathbf{E}^{\text {cont }}=\Delta \mathbf{E}^{\text {direct }}+\Delta \mathbf{E}^{\text {amp }}$.

\section{Data}

Here we consider a subset of the UK banking system composed of the banks that have been consistently part of the concurrent stress test carried out by the Bank of England. ${ }^{10}$ Those banks provide approximately $80 \%$ of the regulated lending to the UK real economy (Bank of England, 2017). From (4) we can see that, in order to compute the fixed point for the vector of equity, one needs external assets and liabilities of all banks, the matrix of interbank assets, and possibly the additional parameters on which valuation functions depend.

\subsection{Balance sheets}

We use two different sources for interbank assets. The first is regulatory data on large exposures that banks have to their counterparties. Banks were required to calculate and report the maximum loss that they would suffer if a counterparty (or group of connected counterparties) failed to meet their obligations. A "large exposure" was defined as any exposure that exceeded $10 \%$ of the bank's capital resources (total Tier 1 and Tier 2 capital after deductions). Banks were prohibited from having exposures greater than $25 \%$ of capital resources (Basel Committee on Banking Supervision, 2014), although exceptions were made for certain counterparties. Due to the aforementioned threshold, these data will tend to underestimate interconnectedness. Nevertheless, they represent the only data about mutual exposures between UK banks that go back until before the peak of the crisis. We can see that, while this entails that the exposures reported are likely to be the output of some valuation performed by the banks, their definition is consistent with (4), given that the maximum loss is realised precisely when the valuation function is equal to zero. Large exposures were collected every quarter from 2008 Q2 to 2013 Q4. The second data source covers every quarter in the period from 2014 Q4 to 2016 Q4. It includes exposures between those seven banks across several asset classes: fixed income (unsecured and subordinated debt securities), securities financing transactions (repo, reverse repo, securities lending and borrowing), and derivatives. In

\footnotetext{
${ }^{10}$ The banks are: Barclays, HSBC, Lloyds Banking Group, Nationwide Building Society, The Royal Bank of Scotland Group, Santander UK, and Standard Chartered.
} 
order to match the definition of the first dataset as closely as possible, we aggregate across all asset classes. In contrast to the "large exposures" data there is no reporting threshold, which allows us to have a more complete picture of the network.

The matrix of interbank liabilities is obtained by transposing the matrix of interbank assets. External liabilities are equal to the difference between total liabilities and interbank liabilities. Total liabilities are obtained from banks' published accounts in their annual and interim reports. Analogously, external assets are equal to the difference between total assets and interbank assets, also obtained from the annual and interim reports. The starting level of equity is computed as total assets minus total liabilities, i.e. through (1), which corresponds to the accounting definition of equity. As a consequence, while the time series for interbank assets and liabilities is updated quarterly, the time series for external assets and liabilities and for initial equities is updated only half-yearly.

In figure 1 we show initial equities, totals assets, and leverage (total assets divided by initial equities) for all banks and throughout the whole period under consideration. Moreover (in four bottom panels), we show average, minimum, and maximum of some network metrics computed from interbank exposures, where we highlight the gap between the two datasets. The out-degree of bank $i$ is the number of its creditors, while its in-degree is the number of its debtors. Those can be taken as simple measures of how interconnected the banking system is. We can see that in the period relative to the first dataset (with a threshold on exposures) the number of counterparties declines, which might be due to some exposures falling below the reporting threshold. In the period relative to the second dataset (without a threshold on exposures) the banking system is almost fully-connected. ${ }^{11}$ However, degrees do not account for the size of interbank exposures. The out-strength of bank $i$ is simply $\sum_{j} A_{i j}(t)$, i.e. the sum of its interbank assets and it is the simplest network metric that accounts for the size of interbank exposures. A network metric specific to financial networks is the interbank leverage (see e.g. Bardoscia et al. (2015, 2017)). The interbank leverage of bank $i$ is equal to $\sum_{j} A_{i j}(t) / E_{i}(t)$, i.e. to the leverage restricted to interbank assets. Both interbank assets and interbank leverages consistently decline throughout the period under consideration.

\subsection{Parameters}

The additional parameters needed to compute valuation functions, as we can see from (7) and from (8), are the recovery rates, the forward-looking time horizon, and the volatility of external assets. We choose a homogeneous recovery rate across banks, i.e. $\rho_{i}=\rho, \forall i$. We mainly use either $\rho=0$, which leads to an upper bound on the losses, or $\rho=0.6$. In fact, actual recovery rates of banks are around 0.56 and 0.57 (see e.g.

\footnotetext{
${ }^{11}$ The maximum out-degree and in-degree is equal to $n-1=6$. We also note that the average out-degree is always equal to the average in-degree.
} 

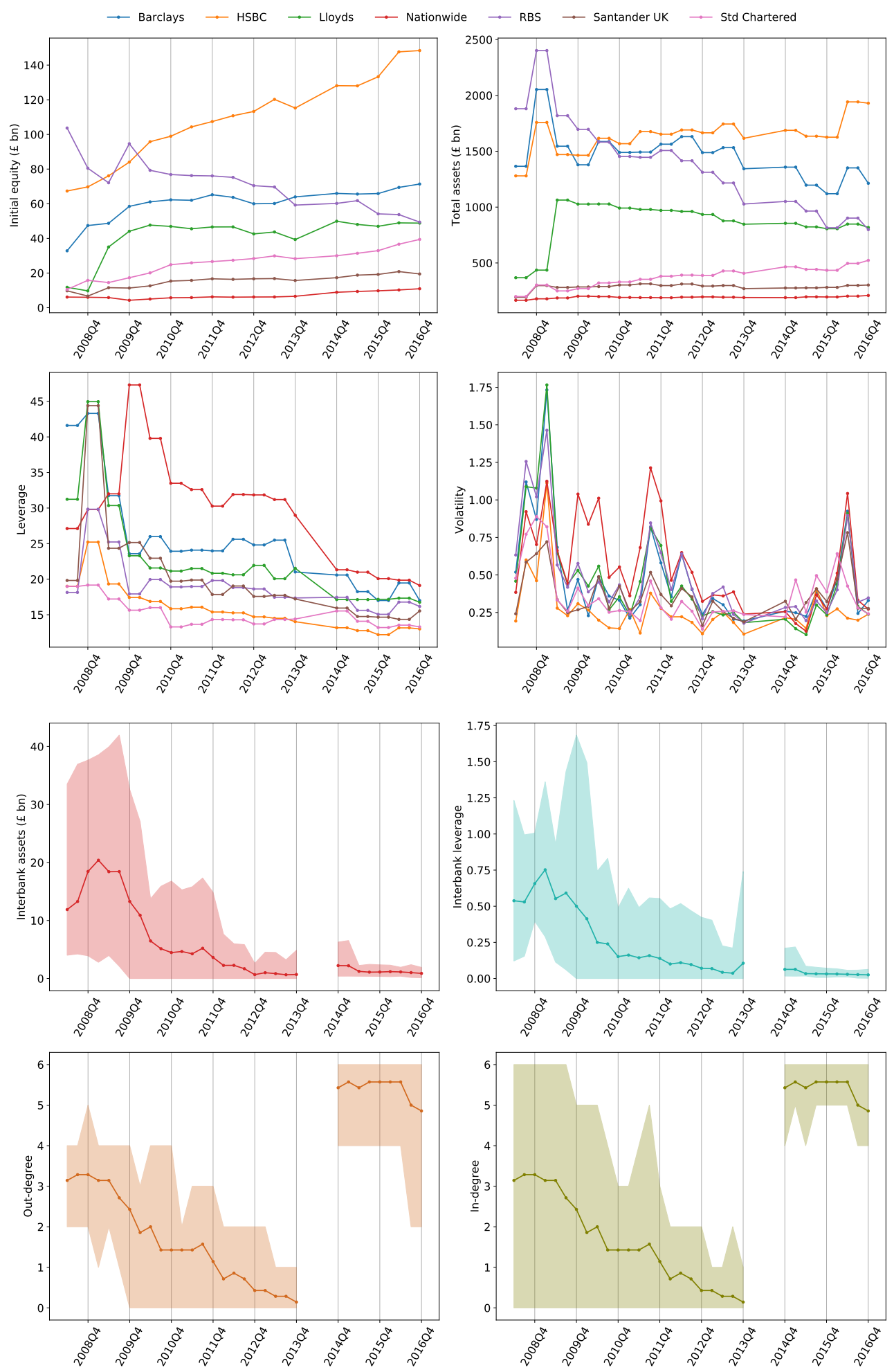

Figure 1: Summary statistics of balance sheets and model parameters. For the four bottom panels solid lines represent the average across banks, while colored regions extend between minima and maxima across banks. The gap in four bottom panels corresponds to the gap between the two datasets used for interbank exposures. 
Jackson and Pernoud (2019) and references therein (James, 1991, Branch, 2002, Acharya et al., 2007, Davydenko et al., 2012)). As an additional sensitivity check, for aggregate contagion losses we scan a broader range of recovery rates.

We fix the forward-looking time horizon $T-t$ to one year in most of our experiments. This is consistent with the time interval between concurrent stress tests run both by the Bank of England and the Federal Reserve. For a sensitivity check on the forward-looking time horizon, see Appendix D. In order to estimate the volatility of external assets, we follow the standard approach used in the Merton model and its generalisations, which consists in assuming that equities follow the same stochastic process as the external assets (albeit with a different volatility). We refer readers interested in the details to Appendix C. Equity volatilities for all banks and throughout the whole period under consideration are shown in figure 1.

\section{Results}

\subsection{Valuation functions}

In figure 2 we plot the calibrated valuation functions for all banks, for 2008 Q3 and 2015 Q1, and for $\rho=0$. In the top panels valuation functions are plotted as functions of equity levels, whereas in the bottom panels they are plotted as functions of equities relative to the initial equity of each bank.

In both cases the right endpoints of all curves correspond to $\mathbf{E}^{\text {pre-shock }}(t)$, the starting (unshocked) values of equities as computed from (1). In absence of equity shocks we have that $\tilde{\mathbf{E}}^{(0)}(t)=\mathbf{E}^{\text {pre-shock }}(t)$. As a consequence, $\mathbb{V}_{j}\left(\tilde{\mathbf{E}}^{(0)}(t)\right)$ is the discount factor that bank $j$ 's creditors initially apply to their interbank assets towards bank $j$, absent any equity shock. We can see that in 2015 Q1 the right endpoint of valuation functions is close to one for all banks. Hence, absent any equity shock, the initial valuation of all interbank assets will be very close to their face value. In contrast, in 2008 Q3 the right endpoint of valuation functions is materially smaller than one for all banks. For example, the right endpoint of the RBS' valuation function is approximately 0.57 , meaning that its creditors initially discount their interbank assets towards RBS by $43 \%$. If equity shocks are non-zero, then $\tilde{\mathbf{E}}^{(0)}(t)=\mathbf{E}^{\text {pre-shock }}(t)-\Delta \mathbf{E}^{\text {shock }}$. Because valuation functions are non-decreasing, any equity shock will push initial valuations of interbank assets further down. Still, in 2015 Q1 most banks would need to suffer a very large shock to their equities to switch into the regime in which valuation functions are materially smaller than one. For example, HSBC would need to lose about $50 \%$ of its initial equity.

Since valuation functions are discount factors for interbank assets, the slopes of curves in the top panel of figure 2 can be interpreted as the additional discount in percentage points applied to interbank assets of a bank hit by an additional equity shock of £1bn. In 2008 Q3 RBS's valuation function is approximately linear, meaning 

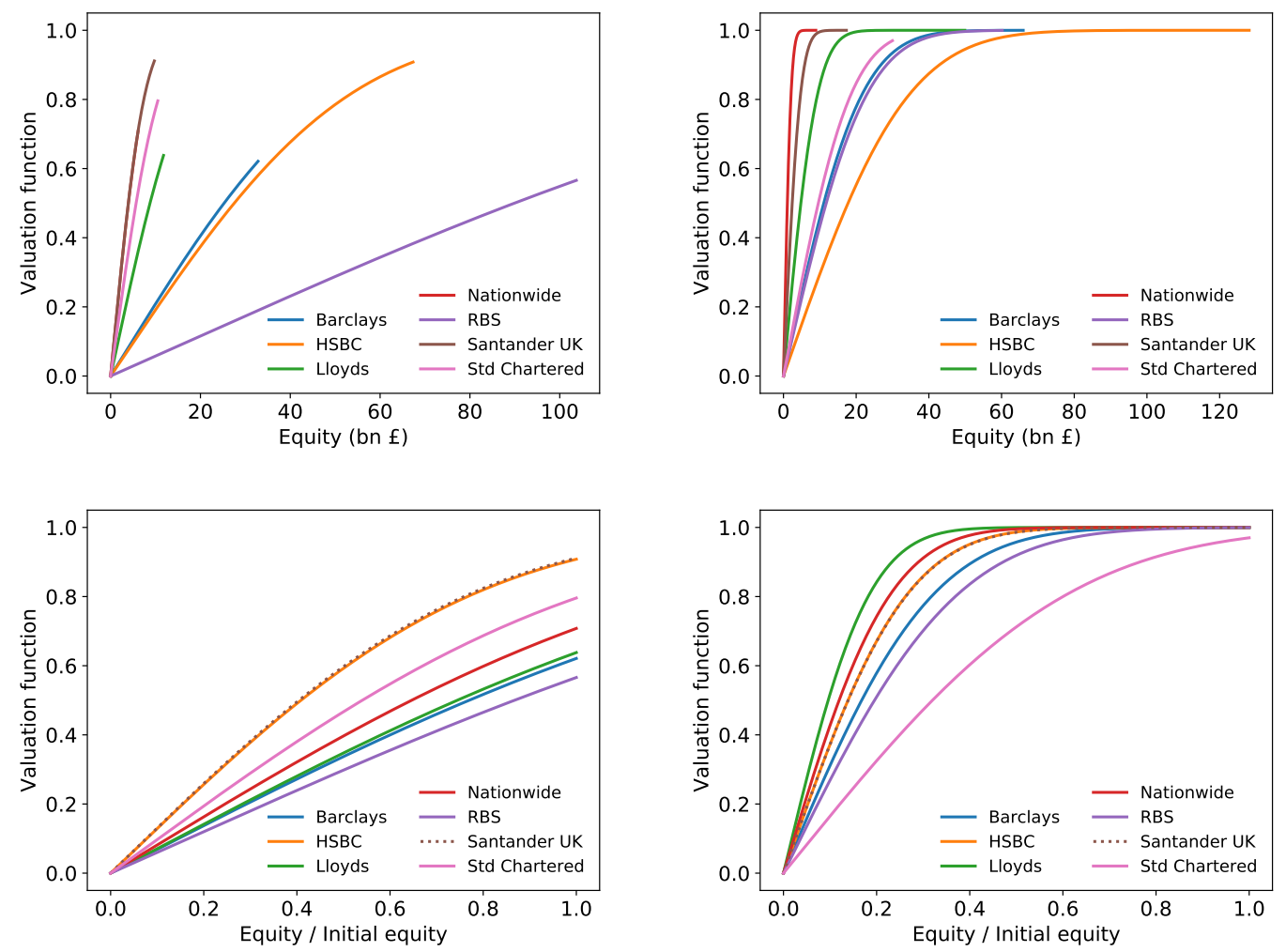

Figure 2: Valuation functions for interbank assets as function of equities (top panels) and as a function of equities relative to initial unshocked equities (bottom panels), both in 2008 Q3 (left panels) and in 2015 Q1 (rights panel), $\rho=0$. The parameters of valuation functions have been calibrated by using data from banks' annual reports and by estimating volatilities of banks' equities from market prices of their stocks.

that any additional equity shock of $£ 1$ bn (about $1 \%$ of its initial equity) translates into an additional devaluation of the interbank assets held by its creditors of about 55 basis points. Lloyds is in a similar situation and any additional equity shock of $£ 1$ bn (about $8.5 \%$ of its initial equity) corresponds to an additional devaluation of the interbank assets held by its creditors of about 542 basis points. In 2015 Q1 for most banks the slope of valuation functions is close to zero for a large range of equity shocks. For example, even if HSBC suffered an exogenous equity shock of $£ 75$ bn (about $50 \%$ of its initial equity) the slope of its valuation function would be still small, meaning that to any additional equity shock of $£ 1$ bn it would correspond a very small additional devaluation of the interbank assets held by its creditors.

Moreover, from figure 2 we can see that valuation functions are generally concave. This means that, for a given additional equity shock, the marginal variation in the discount factor applied to interbank assets is larger when that additional shock is applied to smaller initial equities. This is also in line with the intuition that the impact of a shock on a well-capitalised bank will be smaller than the impact of the same shock on 

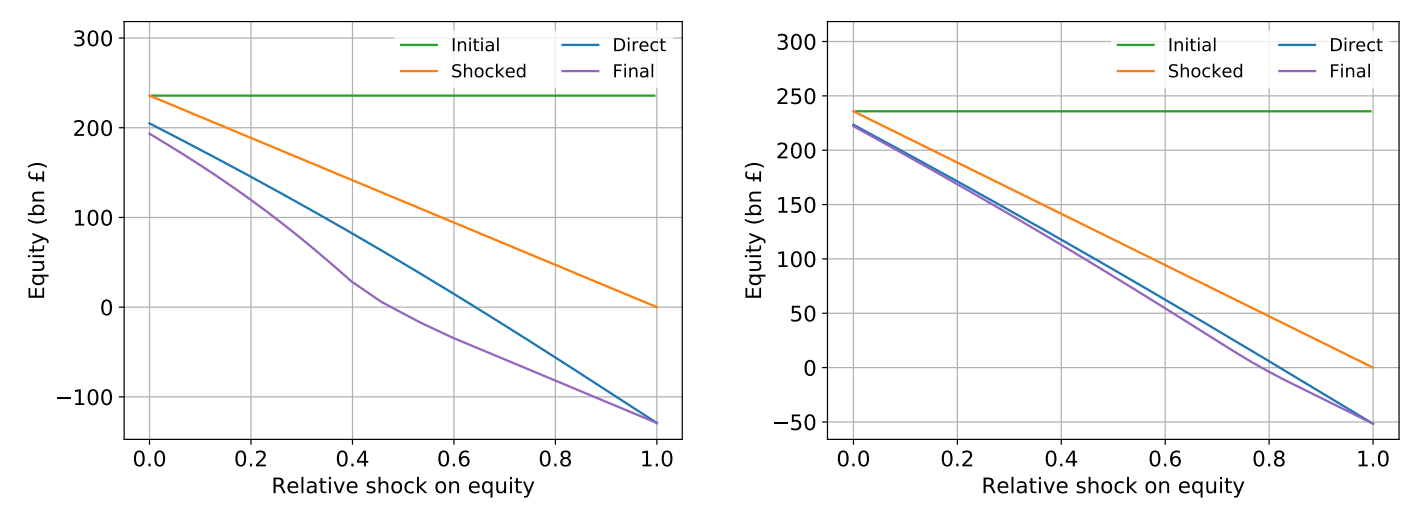

Figure 3: Break down of aggregate losses for a range of homogeneous shocks across banks. Balance sheets and exposures from 2008 Q4, recovery rate $\rho=0$ (left) and $\rho=0.6$ (right).

a poorly-capitalised bank.

Finally, from the bottom panels of figure 2 we can see how different banks respond to the same relative equity shock. When all banks are subject to the same relative equity shock, the ranking of valuation functions from top to bottom corresponds to ranking banks from the ones whose creditors will discount their interbank assets towards them the least to those whose creditors will discount their interbank assets towards them the most.

\subsection{Aggregate contagion losses}

In the remainder of section 4 we carry out simplified stress test exercises. This use of the model can be thought of as a macro-prudential "overlay" on top of a micro-prudential stress test. The initial losses firms suffer should be interpreted as the results of the individual banks' stress test that do not include losses due to contagion. We then use our model to compute the additional losses due to contagion within the system.

We start by exploring the effect of a broad range of shocks. We shock external assets such that the corresponding relative loss of equity $\Delta E_{i}^{\text {shock }} / E_{i}^{\text {pre-shock }}(t)$ is the same across all banks. In figure 3 we break down the different contributions in (10) to total losses for 2008, for two different values of the exogenous recovery rate $\rho$. The green line is simply the aggregate initial equity $\sum_{i} E_{i}^{\text {pre-shock }}(t)$. For a given value of the shock, the distance between the green and the orange line corresponds to the aggregate exogenous shock $\sum_{i} \Delta E_{i}^{\text {shock }}$, the distance between the orange and the blue line corresponds to the aggregate losses caused by direct exposures $\sum_{i} \Delta E_{i}^{\text {direct }}$, while the distance between the blue and the purple line corresponds to the aggregate losses caused by additional rounds of contagion $\sum_{i} \Delta E_{i}^{\text {amp }}$. For $\rho=0$ losses due to contagion can be as large as losses due to the exogenous shock. For $\rho=0.6$, while smaller, they are still sizeable. From figure 3 we also see that there are losses due to contagion 

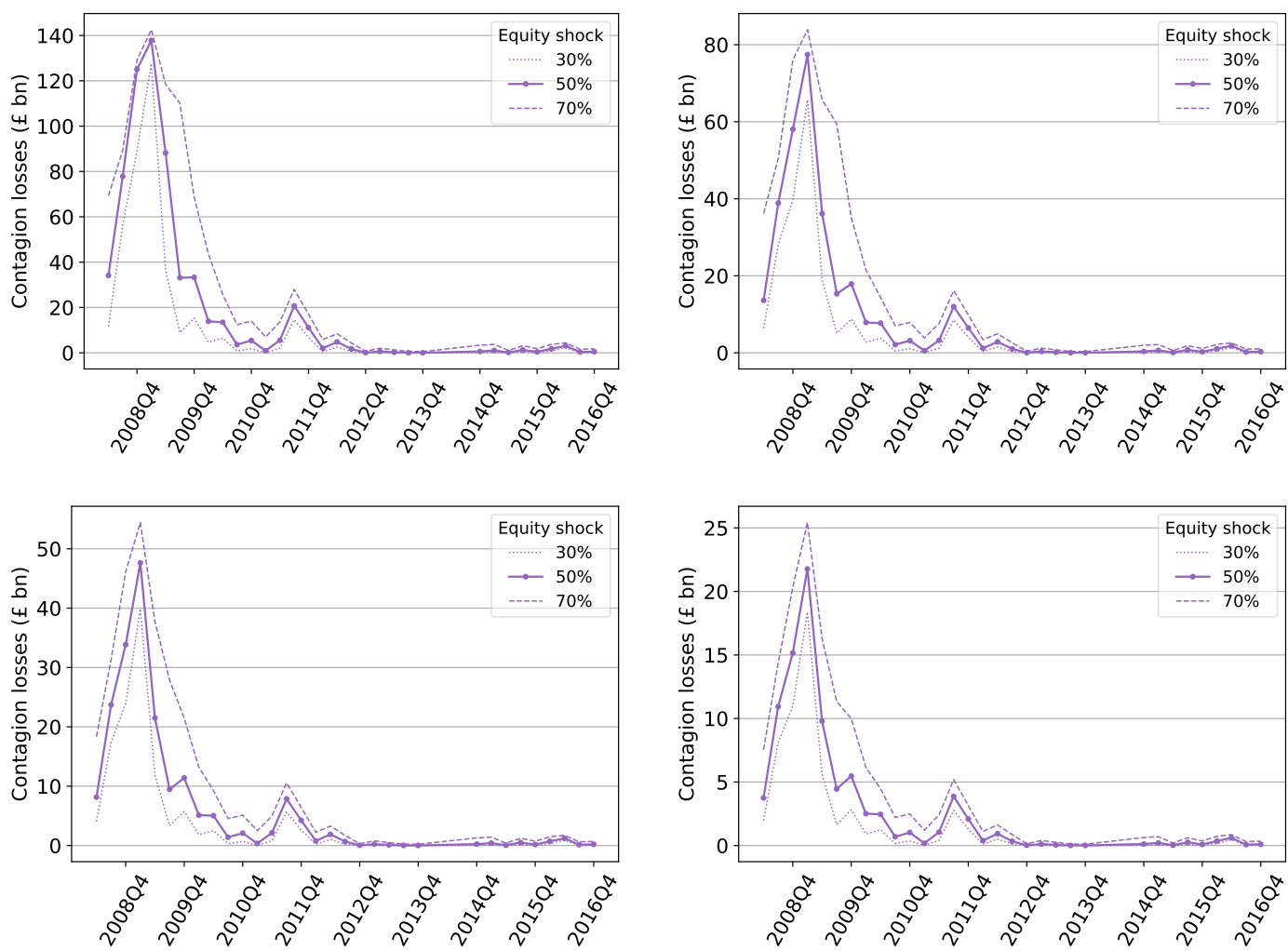

Figure 4: Aggregate losses due to contagion from 2008 to 2016. Relative equity shocks are the same for all banks $(30 \%, 50 \%, 70 \%)$, recovery rates are $\rho=0$ (top left), 0.4 (top right), 0.6 (bottom left), and 0.8 (bottom right).

even without any exogenous shock to equities. This happens because, the valuation of interbank assets in the absence of a shock does not necessarily correspond to the face value of interbank assets, i.e. $\mathbb{V}_{j}\left(E_{j}^{\text {pre-shock }}(t)\right)$ might be strictly smaller than one, as for example in the left panel of figure 2.

Figure 4 shows that aggregate losses due to contagion (corresponding to the difference between the orange line and the purple line in figure 3) decreased significantly from 2008 to 2016 . This result is qualitatively robust to changes of the equity shock or of the recovery rate. It is worth reminding the reader that from 2008 to 2013 our dataset contains only exposures that exceed $10 \%$ of bank's capital and that, as a consequence, across this period exposures - and hence the effect of contagion - might be underestimated. However, even where we use a more granular dataset, from 2014 to 2016, the contagion losses increase only marginally, suggesting that the overall trend in contagious losses is not an artefact of the aforementioned threshold.

In order to better gauge the size of aggregate contagion losses in figure 5 we measure them relative to (aggregate) initial equity shock. In the period between 2008 and 2009 contagion losses can be as large as, or even larger than, the shock in initial equity. As the initial equity shock increases, contagion losses will increase as well, when measured in 

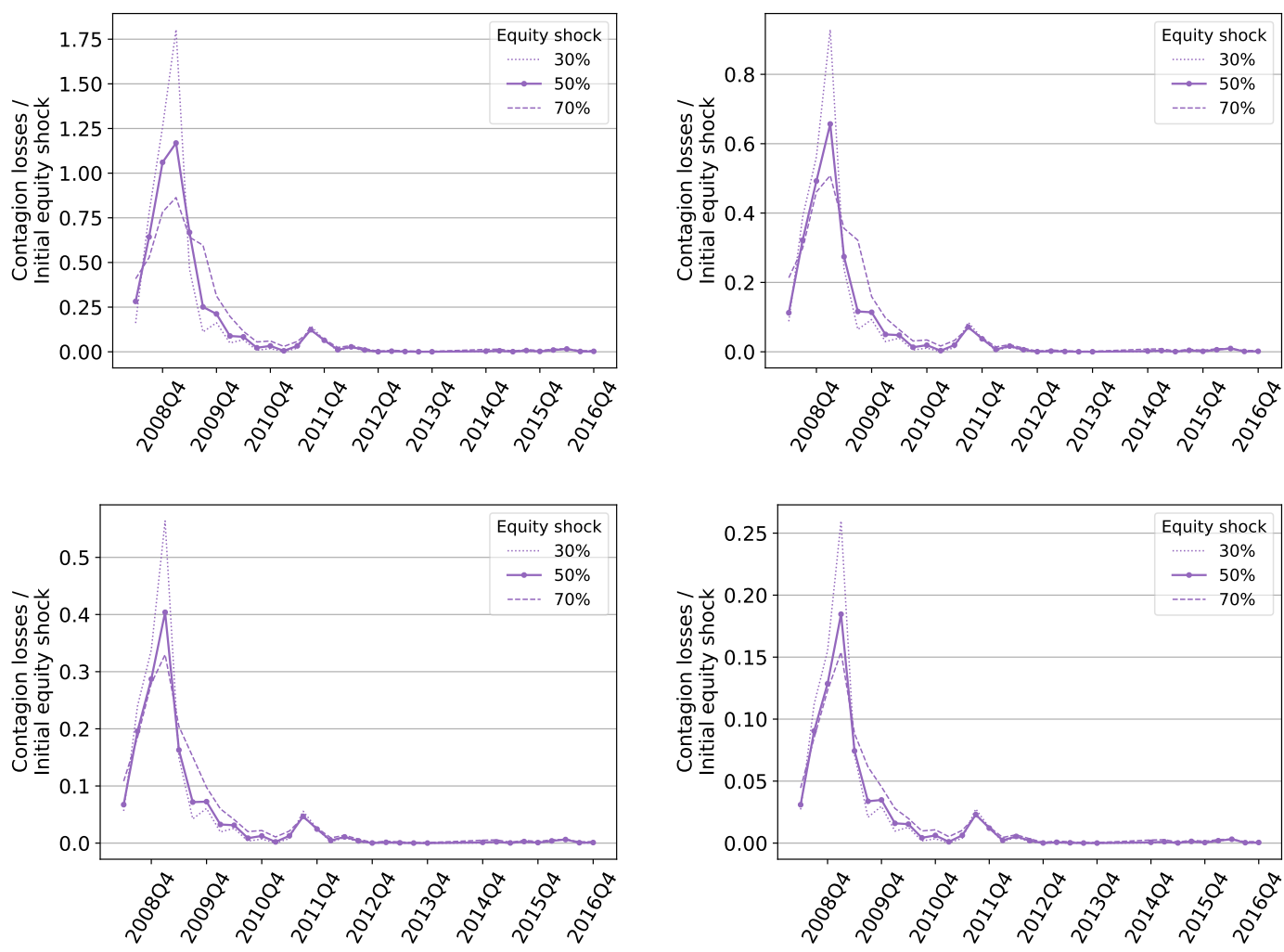

Figure 5: Aggregate losses due to contagion from 2008 to 2016 relative to the initial equity shock. Relative equity shocks are the same for all banks $(30 \%, 50 \%, 70 \%)$, recovery rates are $\rho=0$ (top left), 0.4 (top right), 0.6 (bottom left), and 0.8 (bottom right).

absolute terms. However, when measured relative to the initial equity shock, contagion losses might either increase or decrease, depending on whether they dependence on the initial equity shock is super-linear or sub-linear. This happens indeed in the period between 2008 and 2009, when smaller shocks imply larger contagion losses measured relative the to initial equity shock.

We now compare the exogenous Black and Cox model, on which all the results hitherto presented were based on, with the exogenous Merton model and with the endogenous Merton model. We remind the reader that models without ex-ante uncertainty, i.e. models based on valuation functions of the form (3b), such as Eisenberg and Noe (2001), yield contagion losses equal to zero unless the relative equity shock is larger than $100 \%$ (see section 2.3). By comparing figure 4 with figure 6 , we can see that the endogenous Merton model yields markedly smaller aggregate contagion losses than all the models with exogenous recovery rates. Furthermore, at a given recovery rate, models with default dynamics à la Merton yield smaller aggregate contagion losses than the model with default dynamics à la Black and Cox. This in indeed expected since, as explained in section 2.2, banks default more easily with default dynamics à la Black 

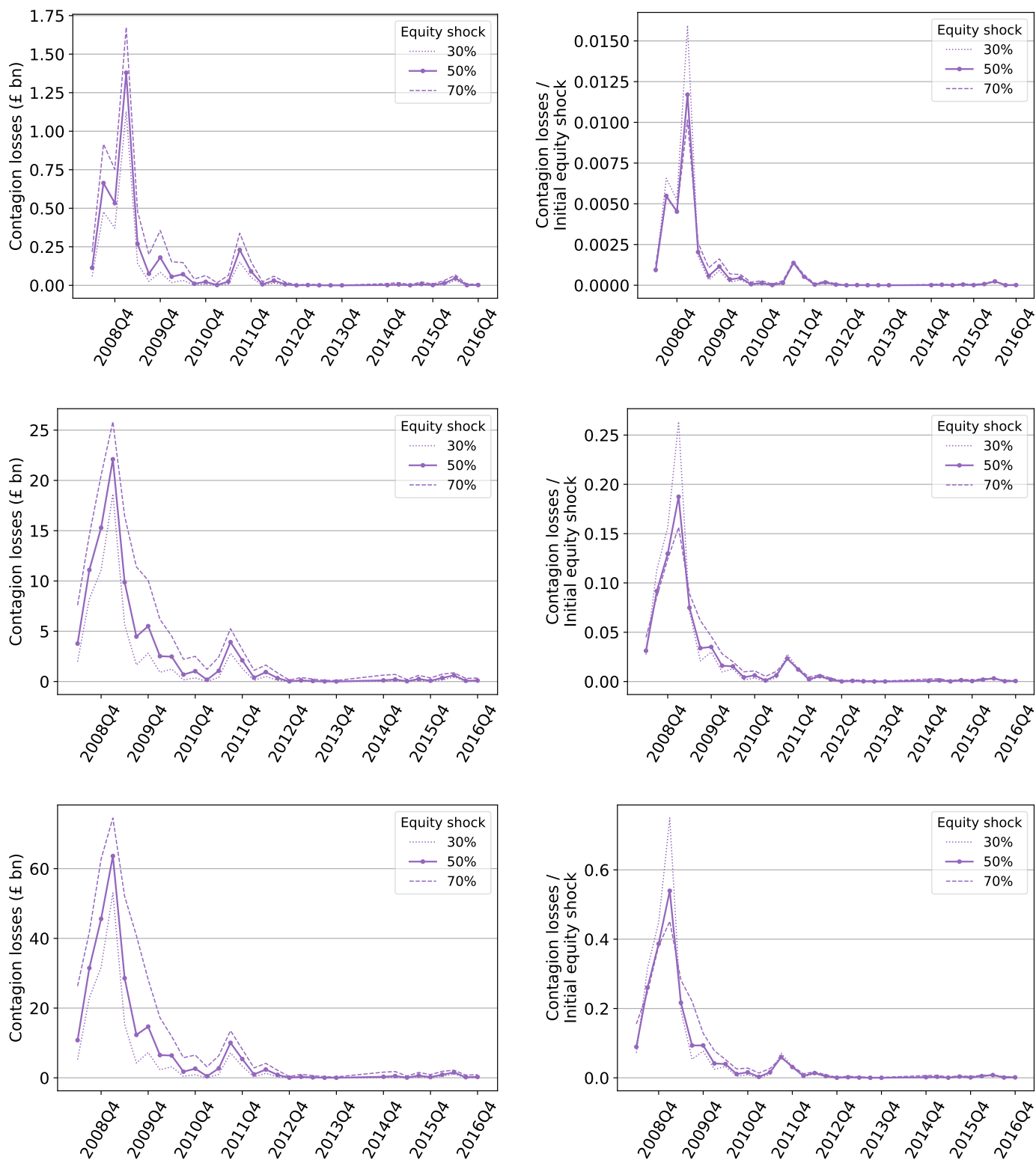

Figure 6: Aggregate losses due to contagion from 2008 to 2016 both in absolute terms (left panels) and relative to the initial equity shock (right panels) for different models. From top to bottom: endogenous Merton model, exogenous Merton model with $\rho=0.6$, exogenous Merton model with $\rho=0$.

and Cox. From figures 5 and 6 we can see that similar results hold for contagion losses when measured relative to the initial equity shock.

In the following subsection we analyse how contagion losses are distributed across banks. The share of contagion losses of a bank will tell us how vulnerable that bank is, while its individual contribution to contagion losses will tell us how systemically important it is. 


\subsection{Distribution of contagion losses}

We now analyse how aggregate contagion losses are distributed across banks. In order to do so, for bank $i$ we define its share of contagion losses:

$$
c_{i}=\frac{\Delta E_{i}^{\mathrm{cont}}}{\sum_{i} \Delta E_{i}^{\mathrm{cont}}}
$$

and we rank them from the largest to the smallest, so that $c_{[1]}$ is the largest share of contagion losses, $c_{[2]}$ is the second largest, and so on. We then define the $k$-th cumulative share of contagion losses as:

$$
C_{0}=0, \quad C_{k}=\sum_{i=1}^{k} c_{[i]} \quad \text { for } k>0 .
$$

$C_{k}$ is simply the sum of the $k$-th largest shares of contagion losses. For example, $C_{1}=0.4$ means that the bank with the largest share of contagion losses is contributing $40 \%$ of the aggregate contagion losses. $C_{2}=0.6$ means that the banks with the two largest share of contagion losses are contributing $60 \%$ of the aggregate contagion losses. A simple way to visualise how contagion losses are concentrated is to plot the cumulative shares of contagion losses $C_{0}, C_{1}, C_{2}, \ldots, C_{n}$ against the fraction of banks $0,1 / n, 2 / n, \ldots, 1$. In fact, if contagion losses are equally distributed across all banks, i.e. $c_{i}=1 / n, \forall i$, from (12) we have that $C_{k}=k / n, \forall k$. As a consequence, the curve of cumulative shares of contagion losses is a straight line with slope equal to one and intercept equal to zero. Conversely, if all contagion losses are concentrated in one bank, we have that $c_{[1]}=1$ and $c_{[i]}=0, \forall i>1$. Hence, while $C_{0}=0$, for $k>1$ we have that $C_{k}=1$ and the curve of cumulative shares of contagion losses is a straight line. In between those two extreme cases, the more concave the curve, the more concentrated the contagion losses are. In this respect it is possible to draw an analogy between a curve of cumulative shares of contagion losses and the inverse of a Lorenz curve representing the distribution of income (or wealth). ${ }^{12}$

In figure 7 we plot the cumulative shares of contagion losses for a selection of periods in our data. We can see that the distribution of contagion losses differs from period to period. In 2009 Q2 about $80 \%$ of contagion losses are split approximately evenly among three banks and the remaining $20 \%$ among four banks. The situation appears to be markedly different in 2011 Q2, when one bank accounts for about $65 \%$ of contagion losses, while the remaining losses are split approximately evenly among five banks. In 2012 Q2 only four banks have material shares of contagion losses, while in 2015 Q4 those are more evenly distributed.

\footnotetext{
${ }^{12}$ In both cases the curves corresponding to zero concentration are straight lines with slope equal to one and intercept equal to zero. However, the more convex a Lorenz curve is, the more unequal the distribution is.
} 

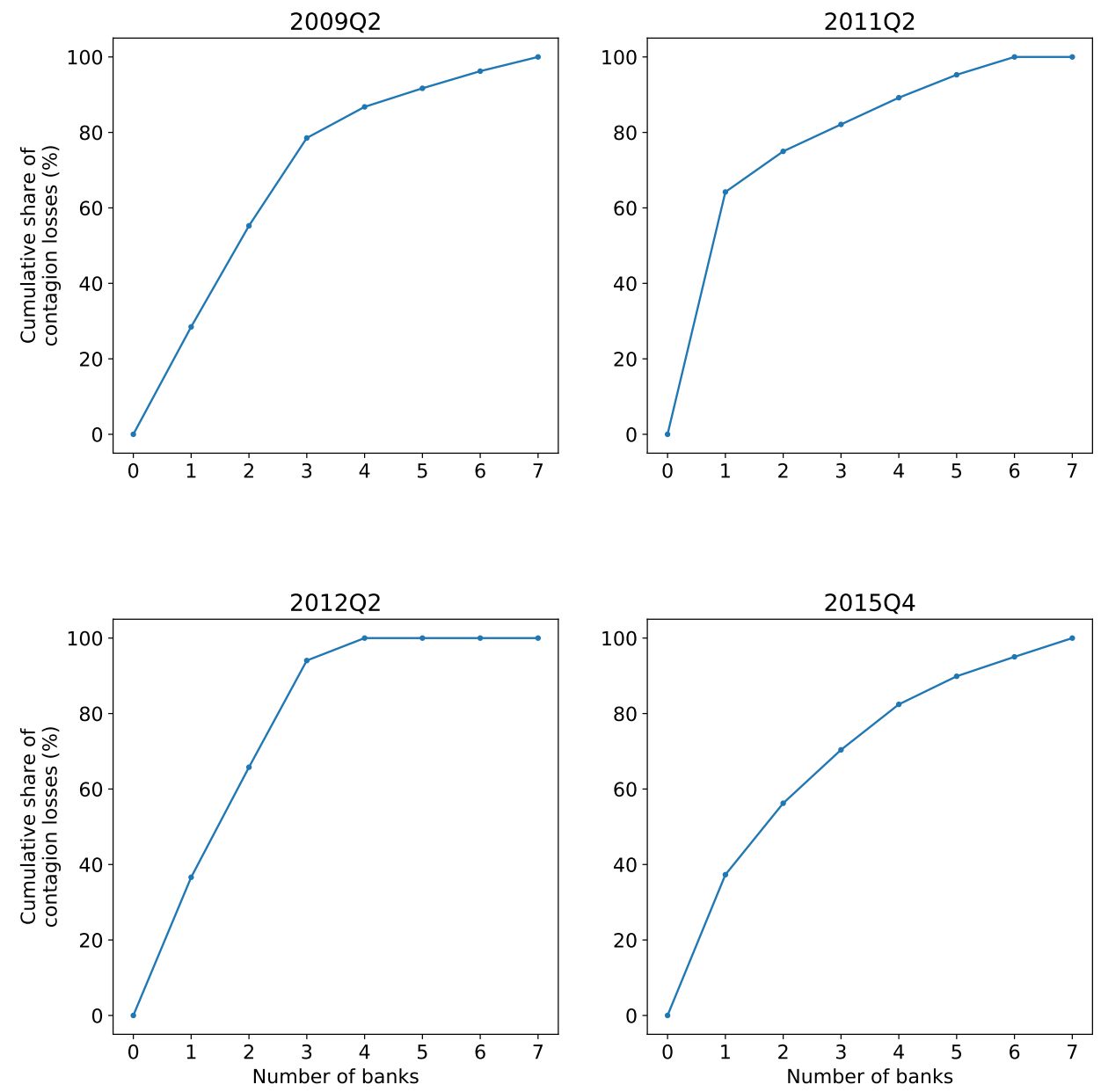

Figure 7: Cumulative shares of contagion losses for a selection of periods in our data. Relative equity shocks are $50 \%$ for all banks, recovery rate $\rho=0$. To enhance readability on the $x$ axis we show the number of banks rather than the fraction of banks.

The share of contagion losses of one bank tells us how vulnerable that bank is relative to the other banks in system, but it does not tell us anything about how dangerous that bank is. In other words, it is a measure of the percentage of losses that a bank might suffer, but not of its systemic importance, which is related to the losses that it is responsible for. A simple way to quantify the marginal contribution of bank $i$ is to compute the difference between the aggregate contagion losses of the banking sub-system that does not include bank $i$, i.e. $\mathcal{B} \backslash\{i\}$ and the aggregate contagion losses of the full banking system $\mathcal{B}$. We denote such difference with $\phi_{i}=\sum_{i} \Delta E_{i}^{\text {cont }}(\mathcal{B} \backslash\{i\})-\sum_{i} \Delta E_{i}^{\text {cont }}(\mathcal{B})$. Banking sub-systems are built simply by converting to external assets all interbank assets corresponding to interbank liabilities of the bank removed and by converting to 
external liabilities all interbank liabilities corresponding to interbank assets of the bank removed. The removal of one bank here can be quite easily interpreted as that bank never being part of the banking system in the first place, or also as that bank defaulting and being liquidated so that all their counterparties recover the full value of their interbank assets, which are then reinvested as external assets. In analogy with the share to contagion losses, we can then define the contributions to contagion losses of bank $i$ :

$$
s_{i}=\frac{\phi_{i}}{\sum_{i} \phi_{i}}
$$

and the corresponding cumulative contributions to contagion losses $S_{0}, S_{1}, S_{2}, \ldots, S_{n}$. We explicitly point out that the sum of all contributions to contagion losses is not necessarily equal to the aggregate contagion losses, i.e. in general $\sum_{i} \phi_{i} \neq \sum_{i} \Delta E_{i}^{\text {cont }}$. This problem can be addressed by following the approach used in Tarashev et al. (2010) and adopted also in Gauthier et al. (2012), Huang et al. (2012), i.e. by performing a Shapley decomposition of contagion losses (see Appendix E). Results are completely analogous to those obtained by using the contributions to contagion losses $\phi_{i}$.

In figure 8 we plot the cumulative contributions to contagion losses for a selection of periods in our data. Also in this case the distribution of contributions to contagion losses changes from period to period. In 2009 Q2 three banks contribute approximately equally to $70 \%$ of contagion losses, two banks contribute to an additional $20 \%$, while the remaining losses are evenly split between two banks. In 2014 Q4 four banks contribute approximately equally to more than $80 \%$ of contagion losses, while three banks contribute evenly to the remaining contagion losses. In 2015 Q3 contributions are more evenly distributed. In 2015 Q4 three banks contribute approximately equally to about $70 \%$ of contagion losses, while four banks contribute evenly to the remaining contagion losses.

An effective way to track concentration over time is to measure the area under the curves (AUCs) of both cumulative shares of and cumulative contributions to contagion losses. When the concentration is minimal, the curves are straight lines with slope one and intercept zero and the AUC is equal to $1 / 2$. When the concentration is maximal, the curves are straight lines with slope zero and intercept one, except for the first point, which is always $(0,0)$. Therefore, the AUC is equal to $1-1 / 2 n$. However, for convenience, we prefer to normalise the AUC such that its minimal value is zero and its maximal value is one. In this way the AUC becomes analogous to the Gini coefficient for the income (or wealth) distribution.

In figure 9 we show the AUCs for every quarter in our data. A comparison with figure 4 reveals that concentration is maximal when aggregate losses are small, signalling that contagion is limited both in its overall effects and in its extent. However, we point out that such temporary increase in concentration may be an artefact of the reporting threshold that affects interbank exposures up to 2013 Q4. In fact, as interbank exposures 

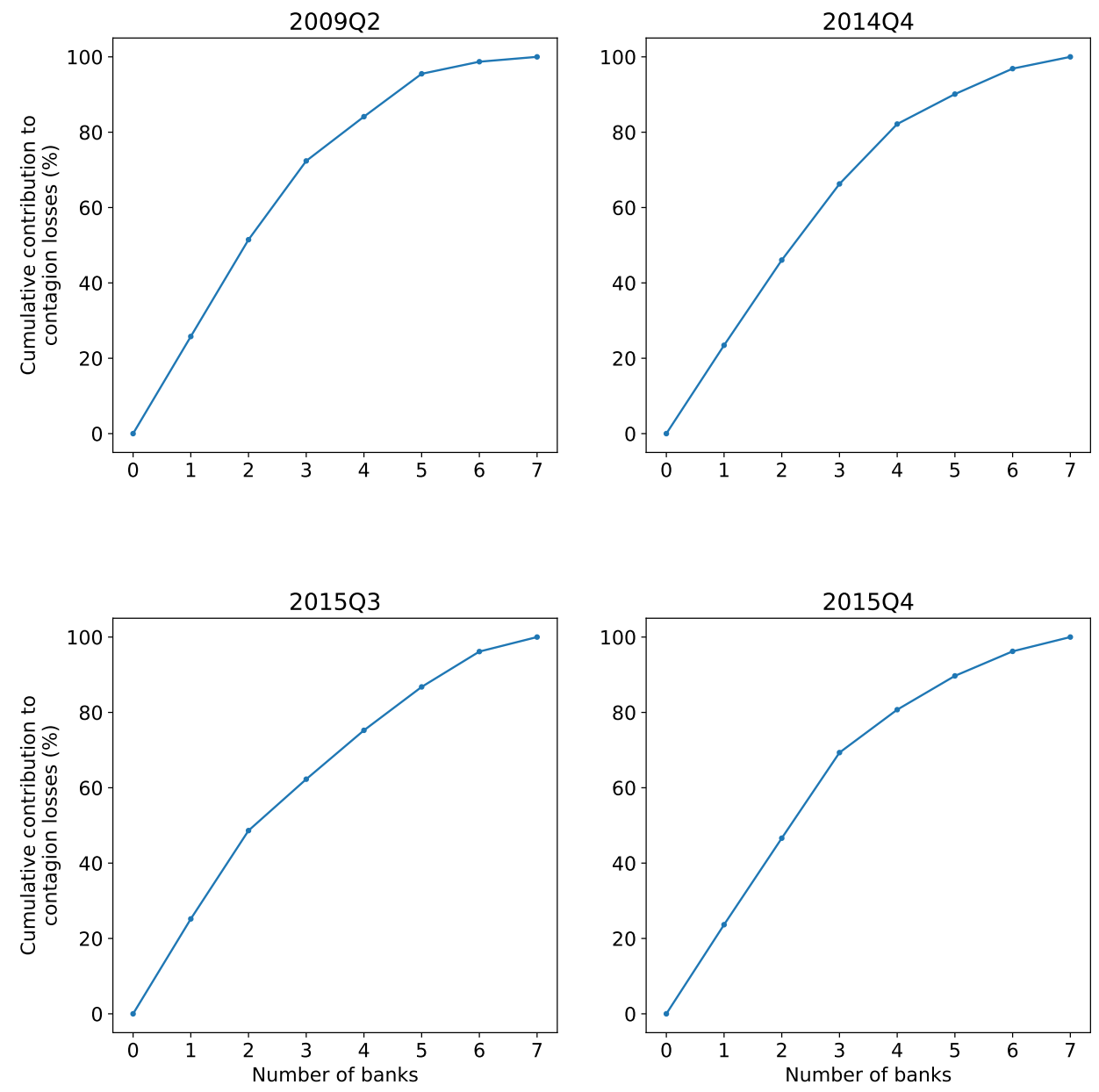

Figure 8: Cumulative contributions to contagion losses for a selection of periods in our data. Relative equity shocks are $50 \%$ for all banks, recovery rate $\rho=0$. To enhance readability on the $x$ axis we show the number of banks rather than the fraction of banks.

decline from 2008 to 2013, only fewer and fewer may have been large enough to be reported. As a consequence, contagion losses would remain confined to the subset of the banking system which is connected by the surviving exposures, naturally resulting in an increase in the concentration of contagion losses. Nevertheless, independently on that observation, from figure 9 we can see that concentrations of both shares of and contributions to contagion losses behave similarly, signalling that vulnerabilities and systemic importances of banks are correlated.

In order to further support this point, in figure 10 we plot shares of contagion losses on one axis and contribution to contagion losses on the other axis, thereby showing 

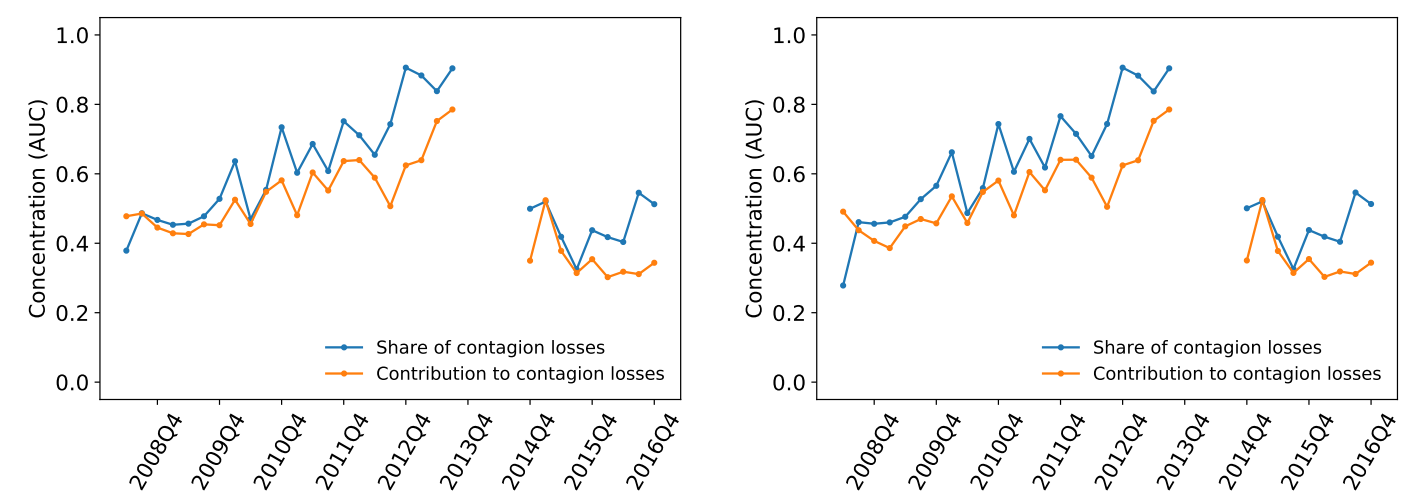

Figure 9: Concentration of shares of and contribution to contagion losses for a selection of periods in our data. The minimal concentration is attained at zero and the maximal at one. Relative equity shocks are $50 \%$ for all banks, recovery rate $\rho=0$ (left) and $\rho=0.6$ (right). The two segments correspond to our two datasets (see section 3).

how those two aspects are interrelated at the level of single banks. Banks lying on the 45 degree line are equally vulnerable and dangerous. Banks below the 45 degree line are relatively more vulnerable than dangerous, and vice versa for banks above the line. The area in bottom right corner corresponds to banks that experience large losses, but that cause small losses. They would be relatively expensive to bail out, and the benefit would be marginal. Conversely, the area in the top left corner corresponds to banks that experience small losses, but that cause large losses. They would be relatively easy to bail out, and the benefit would be large. In practice, in almost all cases banks are fairly close to the 45 degree line and deviate only marginally from it, mostly ruling out such corner cases. We avoid periods corresponding to the peak in figure 9, as in this region the increase in concentration may be an artefact of the reporting threshold on interbank exposures up to 2013 Q4.

\subsection{Bank of England's stress test}

The Bank of England runs an annual concurrent stress test of seven major UK banks. We refer the reader to Bank of England (2015b) for a comprehensive account of the Bank of England's approach to stress testing. The stress test scenario is published every year and it mainly consists of projections of key macroeconomic variables and asset prices. It is designed to capture tail risks and it is calibrated to be countercyclical. This means that, for example, during a housing boom the scenario might include a severe negative shock to house prices. Banks participating in the stress test use their own risk models to project the impact of the scenario on their balance sheets and ultimately on their capital ratios. The Bank of England then uses a range of models and analysis to adjust banks' submissions in order to improve consistency across banks, before the final stress test results are published at the end of the year. 

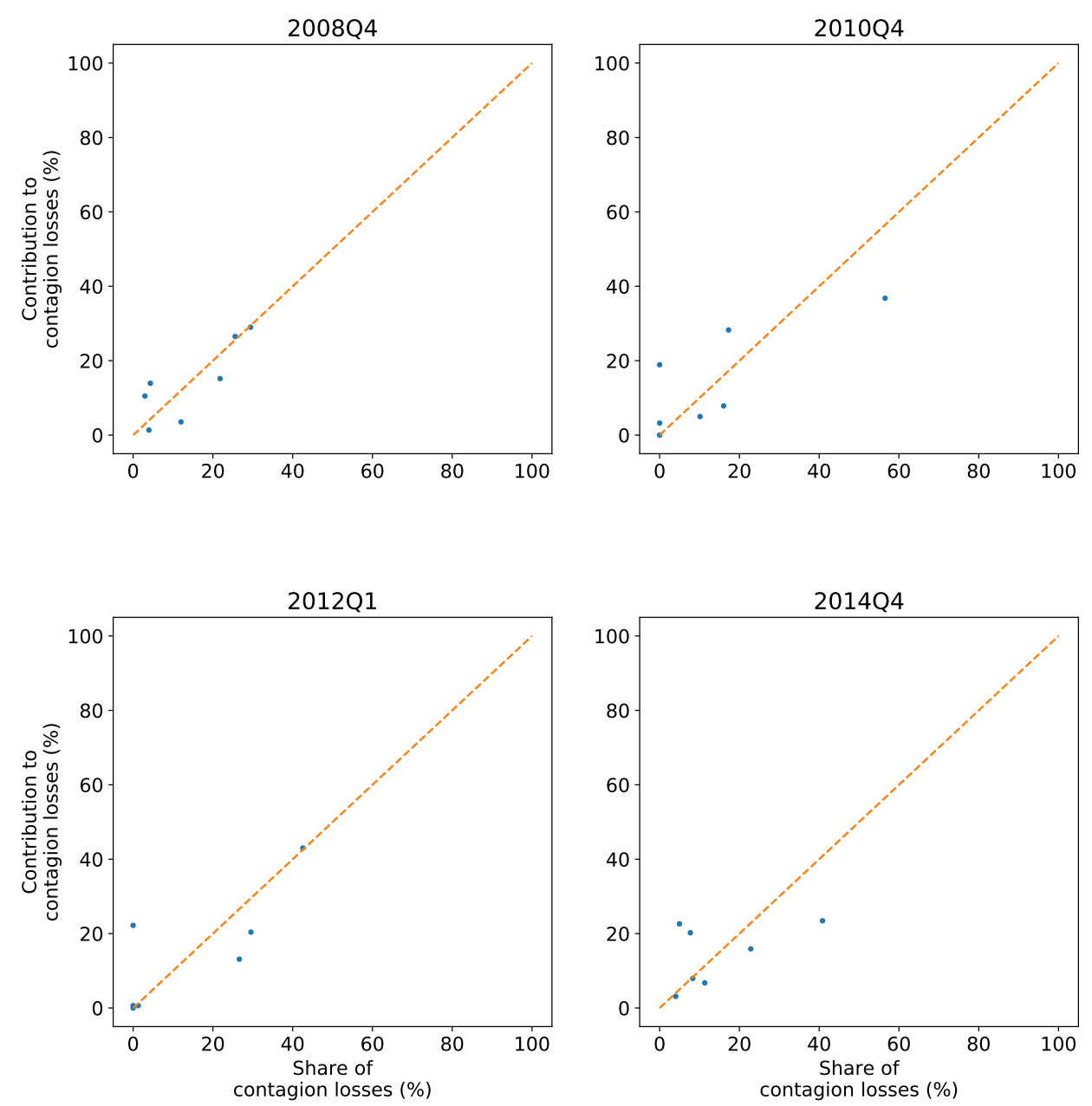

Figure 10: Shares of contagion losses vs contribution to contagion losses for individual banks. Relative equity shocks are $50 \%$ for all banks, recovery rate $\rho=0$.

In this section we take such projected impact on banks' usable capital, which we define as the buffer of regulatory capital that banks hold over and above their capital requirements, as our exogenous shock to banks' equity. We then input this shock to our model to calculate any additional contagion losses. We conduct this exercise for the shocks implied by the Bank of England's concurrent stress tests for the years 2015, 2016 , and 2017.

From 2014, regulators introduced Common Equity Tier 1 (CET1) as the primary measure of regulatory capital, and banks may be put into resolution if their level of CET1 falls below the minimal amount ${ }^{13}$ that regulators require them to hold (Bank of

\footnotetext{
${ }^{13}$ We use the international minimum standard capital requirement as set out in Basel 3, which corre-
} 
England, 2014), i.e. if their usable capital becomes equal to zero. Interpreting the equity of our model as the usable capital is therefore consistent with the fact that in our model banks default as soon their equity becomes equal to zero. In this case external assets must be computed by inverting (1), after the initial usable capital has been specified.

The Bank of England's stess tests are performed using the balance sheets of the final quarter of the previous year as their starting point. In this case it makes perfect sense to use the regulatory interpretation of equity. In fact, each year the stress test consists in assuming a given macroeconomic stress scenario and in projecting the amount of usable capital for each bank under the assumed stress scenario. The starting point, year by year and bank by bank, is the actual value of usable capital, which we interpret as the pre-shock value of equity $\mathbf{E}^{\text {pre-shock }}(t)$. In the stress scenario, usable capital falls both because asset values fall (e.g. banks make credit losses) and this affects capital directly, and because risk-weighted asset rise, which increases a banks' capital requirements. In order to estimate the additional potential losses due to solvency contagion, we use the projected amount of remaining usable capital for each bank envisaged by the results of stress tests as the post-shock equity $\mathbf{E}^{(0)}(t)$ of our model. ${ }^{14}$ More specifically, within the time horizon of the projection, we take the post-shock value of usable capital from the point when each bank is most vulnerable, i.e. when its ratio between CET1 and risk weighted assets is the smallest. ${ }^{15}$

Both the usable capital and the shocks implied by the stress tests for all banks are listed in table 1 . We use $\rho=0$, which allows us to give upper bounds for the case in which interbank exposures are unsecured. We report aggregate contagion losses in table 2. Those are fairly small for all years, reaching a maximum of $3.5 \%$ of the shock to initial equities in 2015 .

\section{Conclusions}

Stress testing has become firmly established as one pillar of the post-crisis regulatory framework. Indeed, it is one of the key tools that regulators employ to set banks' capital. A modern, comprehensive approach to stress testing cannot consider banks in isolation. Instead it should attempt to model the channels through which shocks could propagate from one bank to another, possibly resulting in a systemic crisis. Here we introduce a model for one of those channels, solvency contagion. Importantly, we account not only for losses due to outright defaults of banks, but also for losses due to the revaluation of interbank assets performed by creditors when the probability of

\footnotetext{
sponds to $4.5 \%$ of risk weighted assets Basel Committee on Banking Supervision (2011a).

${ }^{14}$ This method of using the stress test results published by the Bank of England will not be possible in the future, as the stress test results will include losses due to solvency contagion as well.

${ }^{15}$ The values of CET1 and risk weighted assets can be found in the Annex 1 of Bank of England (2015a, 2016, 2017) under the columns "Actual" (pre-shock) and "Minimum stressed ratio (after the impact of 'strategic' management actions)" (post-shock).
} 


\begin{tabular}{lrcc|rcc|rrr}
\cline { 2 - 9 } & \multicolumn{3}{c}{2015} & & \multicolumn{3}{c|}{2016} & \multicolumn{3}{c}{2017} \\
\cline { 2 - 10 } & $\begin{array}{l}\text { Usable } \\
\text { capital } \\
(£ \mathrm{~m})\end{array}$ & Shock & Shock & $\begin{array}{l}\text { Usable } \\
\text { capital } \\
(£ \mathrm{~m})\end{array}$ & $\begin{array}{c}\text { Shock } \\
(\%)\end{array}$ & Shock & $\begin{array}{l}\text { Usable } \\
\text { capital } \\
(£ \mathrm{~m})\end{array}$ & $\begin{array}{c}\text { Shock } \\
(\%)\end{array}$ & Shock \\
\hline Barclays & 22910 & -10890 & -47.5 & 24890 & -9890 & -39.7 & 28530 & -14960 & -52.4 \\
HSBC & 49855 & -20100 & -44.7 & 54520 & -12650 & -23.2 & 63635 & -45441 & -71.4 \\
Lloyds & 20200 & -8090 & -40.0 & 18965 & -5675 & -29.9 & 19280 & -9980 & -51.8 \\
Nationwide & 5635 & 375 & 6.6 & 6425 & -355 & -5.5 & 6470 & -755 & -11.7 \\
RBS & 23980 & -18750 & -78.2 & 27065 & -21540 & -79.6 & 20740 & -13150 & -63.4 \\
Santander UK & 6110 & -1195 & -19.6 & 6130 & -1225 & -20.0 & 6040 & -1135 & -18.8 \\
Std Chartered & 20610 & -10385 & -79.0 & 16820 & -11475 & -68.2 & 20145 & -7370 & -36.6 \\
\hline
\end{tabular}

Table 1: Usable capital and shocks to the usable capital implied by the Bank of England's concurrent stress tests. Both usable capital and shocks have been computed as explained in section 3 using data from the Annex 1 of Bank of England (2015a, 2016, 2017). Years refer to the year in which the stress tests have been carried out. Stress tests are performed by using balance sheets of the final quarter of the previous year.

\begin{tabular}{ccc}
\cline { 2 - 3 } & $\begin{array}{c}\text { Contagion losses } \\
(£ \mathrm{~m})\end{array}$ & $\begin{array}{c}\text { Contagion losses / } \\
\text { Initial equity shock }\end{array}$ \\
\hline 2015 & 2397 & 0.035 \\
2016 & 919 & 0.015 \\
2017 & 1006 & 0.011 \\
\hline
\end{tabular}

Table 2: Contagion losses using shocks implied by the Bank of England's concurrent stress tests (see table 1). We report contagion losses also measured relative to the aggregate shock to initial equities.

default of their counterparties change. The model can be thought as an "overlay" on top of traditional micro-prudential stress test in which banks are stressed in isolation. From this perspective, results of a micro-prudential stress test are exogenous shocks taken as inputs by the model.

By using the model in conjunction with data on bilateral exposures between the seven largest UK banks we show that solvency contagion risk in the UK banking system has declined markedly since the financial crisis. We demonstrate this effect both for homogeneous shocks across all banks and for the shocks implied Bank of England's concurrent stress tests for the years 2015, 2016, and 2017.

We also show how to track the concentration of contagion losses, in terms both of vulnerabilities and systemic importance of individual banks. Classifying banks in terms of those two dimensions could prove especially valuable for regulators. For example, it would allow to identify those banks that are not particularly vulnerable, but that might be highly systemically important, potentially pointing to specific intervention strategies. Finally, the decomposition of the change of contagion losses into key drivers, such as initial equity and in interbank assets, could suggest optimal system-wide policies, such as the implementation of additional capital buffers or capping on interbank exposures. Interestingly, such decomposition might give some indication on whether risks related 
to solvency contagion have genuinely reduced or simply shifted. Loosely speaking, we can imagine that targeting a given expected return will imply a corresponding risk level (or vice versa). Increasing the capital means that, all other things equal, the expected return is mechanically reduced. From this perspective, a negative contribution to the change in contagion losses due to an increase in capital can be interpreted as a genuine reduction of the risk. Conversely, when a bank swaps interbank assets for external assets naturally risk shifts from credit risk to another form of risk that depends on the underlying external assets.

Future research could extend the analysis in two key directions. First, whilst our results show that the risk arising from solvency contagion in the UK does not appear to be substantial at the moment, in reality this risk will interact with other channels of contagion. Such interactions could mutually reinforce effects that would have been small on their own. From a policy perspective, our model should be therefore used within a suite of models that target a wide variety of possible channels of contagion (Halaj, 2018). Second, by its nature, financial system interconnectedness is a global phenomenon, and has the potential to transmit risks across borders. However, we are currently able to conduct analysis only on the subset of the UK interbank network for which we have data. Access to global data through data-sharing initiatives with foreign regulators would facilitate more accurate assessment of the extent of solvency contagion risk facing the UK financial system. For the cases in which exposures would not be available, network reconstruction techniques could be employed to fill the gaps in data.

\section{Acknowledgments}

The authors would like to thank Paul Nahai-Williamson and Amar Radia for their expert advice and Stefano Battiston, Guido Caldarelli, and Fabio Caccioli for countless fruitful discussions. Feedback from Peyton H. Young, Luitgard Veraart, Jose A. Lopez, Camelia Minoiu, Rhiannon Sowerbutts, Matthieu Chavaz, Vesko Karadotchev, Georgina Green, Caterina Lepore, Sinem Hacioglu, and anonymous reviewers has been invaluable and has greatly contributed to improve the manuscript. We are also grateful to a number of participants at seminars organised by the Bank of England for feedback and suggestions. Marco Bardoscia acknowledges previous support from FET Project SIMPOL nr. 610704, FET Project DOLFINS nr. 640772, and H2020 Project CoeGSS nr. 676547. Paolo Barucca acknowledges support from FET Project DOLFINS nr. 640772 and FET IP Project MULTIPLEX nr. 317532. Marco Bardoscia and Paolo Barucca warmly thank the Department of Banking and Finance at the University of Zurich and the London Institute for Mathematical Sciences for their kind hospitality and financial support during the initial stages of the work.

\section{Declarations of interest: none}


Source code. The simulations have been performed by using an implementation of the NEVA framework, which we make available here: https://github.com/marcobardoscia/ neva.

\section{Appendices}

\section{A General model}

Let us now assume that there is a risk-free asset, yielding a (continuously compounded) instantaneous risk-free rate $r$ and that non-stochastic assets and liabilities yield $r$ :

$$
\begin{aligned}
A_{i j}(T) & =e^{r(T-t)} A_{i j}(t) \\
L_{i}(T) & =e^{r(T-t)} L_{i}(t)
\end{aligned}
$$

for all $i$ and $j$, where for brevity we have introduced the total liabilities $L_{i}(s)=L_{i}^{e}(s)+$ $\sum_{j=1}^{n} L_{i j}(s), \forall i, s$. Now $\tilde{\mathbf{E}}(t)$ must include discount factors:

$$
\tilde{\mathbf{E}}(t) \equiv e^{-r(T-t)} \mathbb{E}^{\mathbb{Q}}\left[\tilde{\mathbf{E}}(T) \mid \mathbf{A}^{e}(t)\right]
$$

By plugging (2) in the equation above, we must compute three conditional expectations. For external assets:

$$
e^{-r(T-t)} \mathbb{E}^{\mathbb{Q}}\left[A_{i}^{e}(T) \mid \mathbf{A}^{e}(t)\right]=A_{i}^{e}(t),
$$

because under the measure $\mathbb{Q}$ external assets have a drift term $r \mathrm{~d} s$. For liabilities (interbank plus external):

$$
e^{-r(T-t)} \mathbb{E}^{\mathbb{Q}}\left[L_{i}(T) \mid \mathbf{A}^{e}(t)\right]=e^{-r(T-t)} L_{i}(T)=L_{i}(t)
$$

For interbank assets:

$$
\begin{aligned}
e^{-r(T-t)} \mathbb{E}^{\mathbb{Q}}\left[A_{i j}(T) \mathbb{V}_{j}\left(\tilde{E}_{j}(T)\right) \mid \mathbf{A}^{e}(t)\right] & =e^{-r(T-t)} A_{i j}(T) \mathbb{E}^{\mathbb{Q}}\left[\mathbb{V}_{j}\left(\tilde{E}_{j}(T)\right) \mid \mathbf{A}^{e}(t)\right] \\
& =A_{i j}(t) \mathbb{E}^{\mathbb{Q}}\left[\mathbb{V}_{j}\left(\tilde{E}_{j}(T)\right) \mid \mathbf{A}^{e}(t)\right]
\end{aligned}
$$

and we are left with the conditional expected value of the valuation function to compute.

We start from valuation functions (7), i.e. from the exogenous Black and Cox model. Similarly to the case in which $r=0$, when evaluating bank $j$ 's valuation function, bank $i$ performs the following approximation: $\sum_{k=1}^{n} \tilde{A}_{j k}(s) \approx \sum_{k=1}^{n} \tilde{A}_{j k}(t) e^{r(s-t)}$. In other words, while bank $i$ is still not able to account for the valuation of the interbank assets of bank $j$ 's counterparties, it can correctly track the risk-free increase of those assets. 
Thus, for all $s$ between $t$ and $T, i$ will estimate $j$ 's equity in the following way:

$$
\begin{aligned}
\tilde{E}_{j}(s) & =A_{j}^{e}(s)+\sum_{k} \tilde{A}_{j k}(s)-L_{j}(s) \\
& \approx A_{j}^{e}(s)-L_{j}(s)+e^{r(s-t)} \sum_{k} \tilde{A}_{j k}(t) \\
& =A_{j}^{e}(s)-L_{j}(s)+e^{r(s-t)}\left[\tilde{E}_{j}(t)-A_{j}^{e}(t)+L_{j}(t)\right] \\
& =A_{j}^{e}(s)+\left[\tilde{E}_{j}(t)-A_{j}^{e}(t)\right] e^{r(s-t)} .
\end{aligned}
$$

From (A.6) we can write the survival condition for bank $j$ :

$$
A_{j}^{e}(s) \geq\left[A_{j}^{e}(t)-\tilde{E}_{j}(t)\right] e^{r(s-t)} \quad \forall s \in[t, T] .
$$

If $\tilde{E}_{j}(t)<0$, bank $j$ has already defaulted, and by definition its survival probability must be equal to zero. If $A_{j}^{e}(t) \leq \tilde{E}_{j}(t)$, then (A.7) is always satisfied and the survival probability is equal to one. Hence we focus on the case $0<\tilde{E}_{j}(t)<A_{j}^{e}(t)$. In order to adapt the survival probability (7) in Black and Cox (1976) we re-express our survival condition as $A_{j}^{e}(s) \geq\left[A_{j}^{e}(t)-\tilde{E}_{j}(t)\right] e^{r(T-t)} e^{-r(T-s)}$, which has the same form of the re-organisation boundary in Black and Cox (1976) with $C=\left[A_{j}^{e}(t)-\tilde{E}_{j}(t)\right] e^{r(T-t)}$ and $\gamma=r$. The survival condition also maps into the condition $V(s) \geq K$ in Black and Cox (1976) by taking $V(s)=A_{j}^{e}(s)$ and $K=C$. Finally, $a=0$ in our case, as no dividends are paid. After algebraic manipulations, (7) in Black and Cox (1976) yields (8), which is independent on the value of $r$. Together with (A.3), (A.5), and (A.4), this means that all the equations of the model do not change in the case in which $r>0$.

From (A.7) we can see that the survival condition for bank $j$ depends only on one stochastic process, i.e. $\mathrm{d} A_{j}^{e}(s)$, the stochastic process of bank $j$ 's external assets. As a consequence, the survival probability in (7) and the valuation function itself will not depend on the stochastic process of external assets of any other bank. This means that the valuation function (7) of bank $j$ does not change regardless on whether the stochastic processes of external assets are independent or not. Ultimately, (A.7) is a consequence of the approximation $\sum_{k=1}^{n} \tilde{A}_{j k}(s) \approx \sum_{k=1}^{n} \tilde{A}_{j k}(t) e^{r(s-t)}$, which itself descends from having assumed that banks only have knowledge of their own interbank assets, and therefore that they do not know whether the equity valuations of those counterparties are affected by valuations of interbank assets of those counterparties towards other banks (which would be indirect counterparties for them). This implies that banks are not able to account for potential correlations between external assets.

The case of valuation functions (5), i.e. of the exogenous Merton model is simpler. In this case the survival condition applies only to time $T$ and the approximation analogous to the one made for the exogenous Black and Cox model reads: $\sum_{k=1}^{n} \tilde{A}_{j k}(T) \approx \sum_{k=1}^{n} \tilde{A}_{j k}(t) e^{r(T-t)}$. The corresponding survival condition is: $A_{j}^{e}(T) \geq$ 
$\left[A_{j}^{e}(t)-\tilde{E}_{j}(t)\right] e^{r(T-t)}$. If $A_{j}^{e}(t) \leq \tilde{E}_{j}(t)$, the survival condition is always satisfied and the survival probability is equal to one. For $\tilde{E}_{j}(t)<A_{j}^{e}(t)$, based on the survival condition, the survival probability is simply the survival probability of the standard Merton model with liabilities equal to $C=\left[A_{j}^{e}(t)-\tilde{E}_{j}(t)\right] e^{r(T-t)}$, assets equal to external assets $A_{j}^{e}(s)$, and no dividends. Hence:

$$
\mathbb{E}^{\mathbb{Q}}\left[\mathbb{1}_{\tilde{E}_{j}(T) \geq 0} \mid \mathbf{A}^{e}(t)\right]= \begin{cases}\mathcal{N}\left[\frac{\log \frac{A_{j}^{e}(t)}{A_{j}^{e}(t)-\tilde{E}_{j}(t)}-\frac{\sigma_{j}^{2}(T-t)}{2}}{\sigma_{j} \sqrt{(T-t)}}\right] & \text { for } \tilde{E}_{j}(t)<A_{j}^{e}(t) . \\ 1 & \text { for } \tilde{E}_{j}(t) \geq A_{j}^{e}(t)\end{cases}
$$

The discussion above on the potential correlations between external assets for the exogenous Black and Cox model applies to the exogenous Merton model as well.

\section{B Feasible valuation functions}

Following the definition in Barucca et al. (2016), in order to show that (7) is a feasible valuation function we need to show that: (i) it takes values in the interval [0, 1], (ii) is non-decreasing, and (iii) is continuous from above. In order to prove (i) we simply note that, since $\mathbb{E}^{\mathbb{Q}}\left[\mathbb{1}_{\tau_{j}>T} \mid \mathbf{A}^{e}(t)\right]$ is the expectation of an indicator function, it must be between zero and one. Hence, (7) must be between $\rho_{j}$ and one.

In order to prove (ii) it is sufficient to prove that $\mathbb{E}^{\mathbb{Q}}\left[\mathbb{1}_{\tau_{j}>T} \mid \mathbf{A}^{e}(t)\right]$ is a non-decreasing function of $\tilde{E}_{j}(t)$. Also, we can restrict ourselves to the interval $0<\tilde{E}_{j}(t)<A_{j}^{e}(t)$, in which $\mathbb{E}^{\mathbb{Q}}\left[\mathbb{1}_{\tau_{j}>T} \mid \mathbf{A}^{e}(t)\right]$ is not constant. By introducing the notation $x=\frac{A_{j}^{e}(t)}{A_{j}^{e}(t)-\tilde{E}_{j}(t)}$ and $y=\frac{\sigma_{j}^{2}(T-t)}{2}$ we can re-write (8) in that interval as:

$$
\begin{aligned}
\mathbb{E}^{\mathbb{Q}}\left[\mathbb{1}_{\tau_{j}>T} \mid \mathbf{A}^{e}(t)\right] & =\mathcal{N}\left[\frac{\log x-y}{\sqrt{2 y}}\right]-x \mathcal{N}\left[-\frac{\log x+y}{\sqrt{2 y}}\right] \\
& =\mathcal{N}\left[\frac{\log x-y}{\sqrt{2 y}}\right]-x\left(1-\mathcal{N}\left[\frac{\log x+y}{\sqrt{2 y}}\right]\right) \\
& =\mathcal{N}\left[\frac{\log x-y}{\sqrt{2 y}}\right]+x \mathcal{N}\left[\frac{\log x+y}{\sqrt{2 y}}\right]-x
\end{aligned}
$$

with $x>1$ and $y>0$. We now compute its derivative with respect to $\tilde{E}_{j}(t)$ :

$$
\frac{\partial x}{\partial \tilde{E}_{j}(t)}\left[\frac{1}{\sqrt{2 y} x} \frac{e^{-\frac{1}{2}\left(\frac{\log x-y}{\sqrt{2 y}}\right)^{2}}}{\sqrt{2 \pi}}+\frac{1}{\sqrt{2 y}} \frac{e^{-\frac{1}{2}\left(\frac{\log x+y}{\sqrt{2 y}}\right)^{2}}}{\sqrt{2 \pi}}+\mathcal{N}\left[\frac{\log x+y}{\sqrt{2 y}}\right]-1\right] .
$$

By noting that $\frac{\partial x}{\partial \tilde{E}_{j}(t)}=\frac{A_{j}^{e}(t)}{\left(A_{j}^{e}(t)-\tilde{E}_{j}(t)\right)^{2}}$ is always positive, we have that $\mathbb{E}^{\mathbb{Q}}\left[\mathbb{1}_{\tau_{j}>T} \mid \mathbf{A}^{e}(t)\right]$ 
is an increasing function of $\tilde{E}_{j}(t)$ as long as:

$$
\frac{1}{\sqrt{2 y} x} \frac{e^{-\frac{1}{2}\left(\frac{\log x-y}{\sqrt{2 y}}\right)^{2}}}{\sqrt{2 \pi}}+\frac{1}{\sqrt{2 y}} \frac{e^{-\frac{1}{2}\left(\frac{\log x+y}{\sqrt{2 y}}\right)^{2}}}{\sqrt{2 \pi}}+\mathcal{N}\left[\frac{\log x+y}{\sqrt{2 y}}\right]-1>0,
$$

which, by introducing $z=1 / x$ and after algebraic manipulations reduces to:

$$
\frac{e^{-\frac{1}{2}\left(\frac{-\log z+y}{\sqrt{2 y}}\right)^{2}}}{\sqrt{\pi y}}+\mathcal{N}\left[\frac{-\log z+y}{\sqrt{2 y}}\right]-1>0,
$$

with $0<z<1$. Indeed, we numerically check that the equation above holds in the full range of $z$ and up to values of $y$ sufficiently large to include the largest volatility observed in our sample.

In order to prove (iii) it is sufficient to prove that $\mathbb{E}^{\mathbb{Q}}\left[\mathbb{1}_{\tau_{j}>T} \mid \mathbf{A}^{e}(t)\right]$ is a continuous function of $\tilde{E}_{j}(t)$ from above. Indeed, it is possible to prove that $\mathbb{E}^{\mathbb{Q}}\left[\mathbb{1}_{\tau_{j}>T} \mid \mathbf{A}^{e}(t)\right]$ is continuous, i.e. both from above and from below. From (8) we can see that $\mathbb{E}^{\mathbb{Q}}\left[\mathbb{1}_{\tau_{j}>T} \mid \mathbf{A}^{e}(t)\right]$ can only be discontinuous either in $\tilde{E}_{j}(t)=0$ or in $\tilde{E}_{j}(t)=A_{j}^{e}(t)$. It is immediate to show that $\lim _{\tilde{E}_{j}(t) \rightarrow 0^{-}} \mathbb{E}^{\mathbb{Q}}\left[\mathbb{1}_{\tau_{j}>T} \mid \mathbf{A}^{e}(t)\right]=0$, implying that $\mathbb{E}^{\mathbb{Q}}\left[\mathbb{1}_{\tau_{j}>T} \mid \mathbf{A}^{e}(t)\right]$ is continuous in zero. In order to prove the continuity in $A_{j}^{e}(t)$, we compute the limit $\tilde{E}_{j}(t) \rightarrow A_{j}^{e}(t)^{+}:$

$$
\begin{aligned}
\lim _{\tilde{E}_{j}(t) \rightarrow A_{j}^{e}(t)^{+}} \mathbb{E}^{\mathbb{Q}}\left[\mathbb{1}_{\tau_{j}>T} \mid \mathbf{A}^{e}(t)\right] & =\lim _{z \rightarrow 0^{+}} \mathcal{N}\left[\frac{-\log z-y}{\sqrt{2 y}}\right]-\lim _{z \rightarrow 0^{+}} \frac{1}{z} \mathcal{N}\left[\frac{\log z-y}{\sqrt{2 y}}\right] \\
& =1-\lim _{z \rightarrow 0^{+}} \frac{1}{2 \sqrt{\pi y z}} e^{-\frac{1}{4 y} \log ^{2} z-\frac{y}{4}} \\
& =1,
\end{aligned}
$$

where we have applied L'Hôpital's rule to proceed from the first to the second line.

All the steps above can be replicated to prove that also (5) is a feasible valuation function. Also in this case $\mathbb{E}^{\mathbb{Q}}\left[\mathbb{1}_{\tilde{E}_{j}(T) \geq 0} \mid \mathbf{A}^{e}(t)\right]$ is the expectation of an indicator function, and therefore it must be between zero and one. Hence, also the right-hand-side of (5) must be between $\rho_{j}$ and one, thereby proving (i). From (A.8), since $\mathcal{N}$ is an increasing function of its argument, it is also an increasing function of $\tilde{E}_{j}(t)$, thereby proving (ii). Finally, the $\operatorname{limit} \lim _{\tilde{E}_{j}(t) \rightarrow A_{j}^{e}(t)^{+}} \mathbb{E}^{\mathbb{Q}}\left[\mathbb{1}_{\tau_{j}>T} \mid \mathbf{A}^{e}(t)\right]=1$ readily implies that $\mathbb{E}^{\mathbb{Q}}\left[\mathbb{1}_{\tilde{E}_{j}(T) \geq 0} \mid \mathbf{A}^{e}(t)\right]$ is a continuous function of $\tilde{E}_{j}(t)$, thereby proving (iii).

\section{Volatility of external assets}

In order to estimate the volatility of external assets we adapt the standard approach followed in credit structural models to estimate the volatility of total assets. We assume that (valuations of) equities follow geometric Brownian motion, albeit with different 
drifts and volatilities than external assets, i.e. $\mathrm{d} \tilde{E}_{j}(s)=\mu_{j}^{E} \tilde{E}_{j}(s) \mathrm{d} s+\sigma_{j}^{E} \tilde{E}_{j}(s) \mathrm{d} W_{j}(s)$, $\forall j$. Because from (4) the (valuation of) bank $j$ 's equity depends explicitly on bank $j$ 's external assets it is possible, via Ito's lemma, to derive a general relationship between the volatility of bank $j$ 's equity and the volatility of bank $j$ 's external assets at time $t$ :

$$
\sigma_{j} A_{j}^{e}(t) \frac{\partial \tilde{E}_{j}(t)}{\partial A_{j}^{e}(t)}=\sigma_{j}^{E} \tilde{E}_{j}(t) \quad \forall j
$$

From (4) we see that $\tilde{E}_{j}(t)$ depends explicitly on $A_{j}^{e}(t)$ only through the first term, i.e. $\partial \tilde{E}_{j}(t) / \partial A_{j}^{e}(t)=1$, which implies:

$$
\sigma_{j}=\frac{\tilde{E}_{j}(t)}{A_{j}^{e}(t)} \sigma_{j}^{E} \quad \forall j .
$$

For external assets and equities we use the value implied by the banks' balance sheets, as explained in section 3 , while $\sigma_{j}^{E}$, the volatility of bank $j$ 's equity is estimated for each quarter as follows. We compute the daily log-returns using closing prices of bank $j$ shares for the latest 30 working days of the quarter under consideration. We use closing prices instead of market capitalisations to in order to avoid outliers due to events such as buy-backs. We then compute daily volatilities as the standard deviation of the time series of daily log-returns. Daily volatilities are annualised by multiplying daily volatilities by the square root of the number of working days in one year (250). A notable exception is Nationwide, which is not publicly traded. Given the similar asset portfolio of the two banks, we set the volatility of Nationwide's external assets equal to Lloyds'. The volatility of Nationwide's equity (as shown in figure 1) is then simply obtained by inverting (A.9).

\section{Forward-looking time horizon}

In figure A.1 we explore the role of the forward-looking time horizon $T-t$. By comparing it with figures 4 and 5 we can see that the qualitative behaviour of aggregate contagion losses does not change, both when measured in absolute terms and when measured relative to the (aggregate) initial equity shock. However, a shorter time horizon slightly enhances the differences between peaks and troughs in aggregate contagion losses, while a longer time horizon slightly diminishes such differences.

\section{E Shapley decomposition of contagion losses}

Shapley values are a standard way to attribute quotas of the total payoff to individual players participating in a cooperative game (Shapley, 1953). In order to identify the contribution to contagion losses of one bank with its Shapley value, we consider banks 
to be players in a game whose total payoff is equal to the aggregate contagion losses. In practice, in order to compute $\phi_{i}^{\mathrm{s}}$, the Shapley value of bank $i$, one has to compute the difference between contagion losses of any banking sub-system $\mathcal{S}$ that does not contain bank $i$ and contagion losses of the same subset to which bank $i$ has been added, and to suitably average that difference over all such possible banking sub-systems:

$$
\phi_{i}^{\mathrm{s}}=\sum_{\mathcal{S}: i \notin \mathcal{S}} \frac{|\mathcal{S}| !(|\mathcal{B}|-|\mathcal{S}|-1 \mid !)}{|\mathcal{B}| !}\left[\Delta E_{i}^{\mathrm{cont}}(\mathcal{S})-\Delta E_{i}^{\mathrm{cont}}(\mathcal{S} \cup\{i\})\right],
$$

Remarkably, the sum of all Shapley values is equal to the total payoff, i.e. to aggregate contagion losses. As a robustness check we have computed Shapley values of all banks and checked that the resulting contribution to contagion losses are completely analogous to those obtained by using the definition of contributions to contagion losses in section 4.3. However, because Shapley values involve an average over all the possible banking sub-systems, in our opinion they are not as easily interpretable as the contributions to contagion losses $\phi_{i}$ in section 4.3 , and therefore we only present results relative to the latter. 

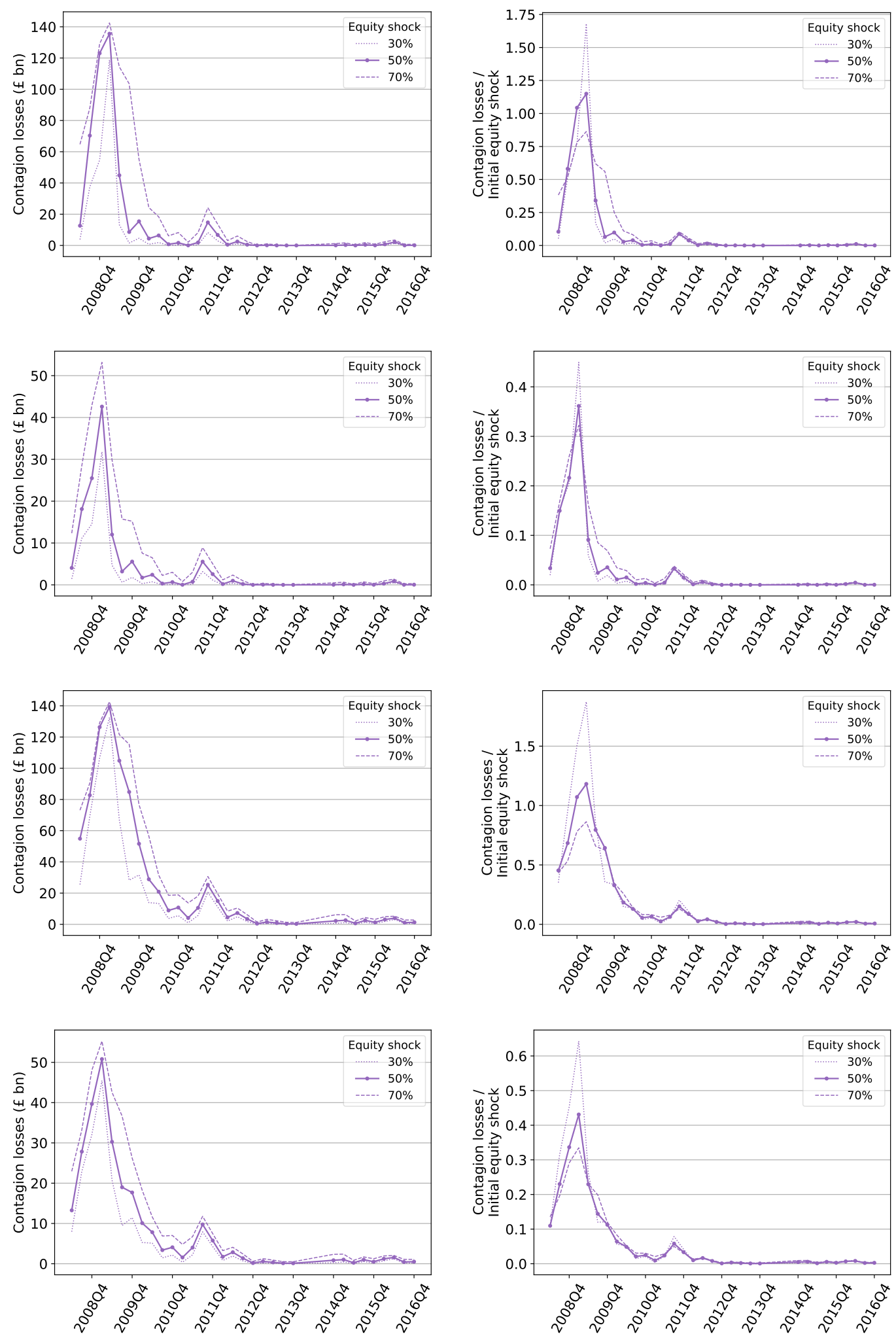

Figure A.1: Aggregate losses due to contagion from 2008 to 2016 both in absolute terms (left panels) and relative to the initial equity shock (right panels) for different forwardlooking time horizons. From top to bottom: $T-t=6$ months and $\rho=0.0, T-t=6$ months and $\rho=0.6, T-t=2$ years and $\rho=0.0$, and $T-t=2$ years and $\rho=0.6$. 


\section{References}

Acemoglu, D., Ozdaglar, A., and Tahbaz-Salehi, A. (2015). Systemic risk and stability in financial networks. The American Economic Review, 105(2):564-608.

Acharya, V. V., Bharath, S. T., and Srinivasan, A. (2007). Does industry-wide distress affect defaulted firms? evidence from creditor recoveries. Journal of Financial Economics, 85(3):787-821.

Acharya, V. V., Pedersen, L., Philippon, T., and Richardson, M. (2013). Taxing systemic risk. In Managing and Measuring Risk: Emerging Global Standards and Regulations After the Financial Crisis, pages 99-122. World Scientific, Singapore.

Adrian, T. and Brunnermeier, M. K. (2016). CoVaR. The American Economic Review, 106(7):1705.

Allen, F. and Gale, D. (2000). Financial contagion. Journal of Political Economy, 108(1):1-33.

Amini, H., Cont, R., and Minca, A. (2013). Resilience to contagion in financial networks. Mathematical Finance, 26(2):329-356.

Anand, K., Craig, B., and Von Peter, G. (2015). Filling in the blanks: Network structure and interbank contagion. Quantitative Finance, 15(4):625-636.

Banerjee, T., Bernstein, A., and Feinstein, Z. (2018). Dynamic clearing and contagion in financial networks. Available from: https://arxiv.org/abs/1801.02091.

Bank of England (2014). The Bank of England's approach to resolution. Available from: http://www.bankofengland.co.uk/financialstability/Documents/ resolution/apr231014.pdf.

Bank of England (2015a). Stress testing the UK banking system: 2015 results. Available from: https://www.bankofengland.co.uk/-/media/boe/files/stress-testing/ 2015/stress-testing-the-uk-banking-system-2015-results .pdf.

Bank of England (2015b). The Bank of England's approach to stress testing the UK banking system. Available from: https: //www.bankofengland.co.uk/-/media/boe/files/stress-testing/2015/ the-boes-approach-to-stress-testing-the-uk-banking-system.

Bank of England (2016). Stress testing the UK banking system: 2016 results. Available from: https://www.bankofengland.co.uk/-/media/boe/files/stress-testing/ 2016/stress-testing-the-uk-banking-system-2016-results .pdf. 
Bank of England (2017). Stress testing the UK banking system: 2017 results. Available from: https://www. bankofengland.co.uk/-/media/boe/files/stress-testing/ 2017/stress-testing-the-uk-banking-system-2017-results.pdf.

Bardoscia, M., Battiston, S., Caccioli, F., and Caldarelli, G. (2015). Debtrank: A microscopic foundation for shock propagation. PloS One, 10(6):e0130406.

Bardoscia, M., Battiston, S., Caccioli, F., and Caldarelli, G. (2017). Pathways towards instability in financial networks. Nature Communications, 8:14416.

Bardoscia, M., Caccioli, F., Perotti, J. I., Vivaldo, G., and Caldarelli, G. (2016). Distress propagation in complex networks: the case of non-linear DebtRank. PloS One, 11(10):e0163825.

Barucca, P., Bardoscia, M., Caccioli, F., D'Errico, M., Visentin, G., Battiston, S., and Caldarelli, G. (2016). Network valuation in financial systems. Available from: https://ssrn. com/abstract=2795583.

Basel Committee on Banking Supervision (2011a). Basel III: A global regulatory framework for more resilient banks and banking systems. Available from: http: //www.bis.org/publ/bcbs189.pdf.

Basel Committee on Banking Supervision (2011b). Capital treatment for bilateral counterparty credit risk finalised by the Basel Committee. Available from: http: //www.bis.org/press/p110601.htm.

Basel Committee on Banking Supervision (2014). Supervisory framework for measuring and controlling large exposures. Available from: http://www.bis.org/publ/ bcbs283.pdf.

Battiston, S., Puliga, M., Kaushik, R., Tasca, P., and Caldarelli, G. (2012). Debtrank: Too central to fail? Financial networks, the fed and systemic risk. Scientific Reports, $2: 541$.

Bielecki, T. R., Jeanblanc, M., and Rutkowski, M. (2004). Modeling and valuation of credit risk. In Stochastic methods in finance, pages 27-126. Springer.

Black, F. and Cox, J. C. (1976). Valuing corporate securities: Some effects of bond indenture provisions. The Journal of Finance, 31(2):351-367.

Branch, B. (2002). The costs of bankruptcy: A review. International Review of Financial Analysis, 11(1):39-57.

Brigo, D. and Tarenghi, M. (2004). Credit default swap calibration and equity swap valuation under counterparty risk with a tractable structural model. Available from: https://ssrn. com/abstract=581302. 
Caccioli, F., Shrestha, M., Moore, C., and Farmer, J. D. (2014). Stability analysis of financial contagion due to overlapping portfolios. Journal of Banking $\mathscr{E}$ Finance, $46: 233-245$.

Cifuentes, R., Ferrucci, G., and Shin, H. S. (2005). Liquidity risk and contagion. Journal of the European Economic Association, 3(2-3):556-566.

Cimini, G., Squartini, T., Garlaschelli, D., and Gabrielli, A. (2015). Systemic risk analysis on reconstructed economic and financial networks. Scientific Reports, 5:15758.

Cont, R., Moussa, A., and Santos, E. B. (2010). Network structure and systemic risk in banking systems. Available from: https://ssrn.com/abstract=1733528.

Cont, R., Moussa, A., and Santos, E. B. (2013). Network structure and systemic risk in banking systems. In Fouque, J.-P. and Langsam, J. A., editors, Handbook on Systemic Risk, chapter 13, pages 327-336. Cambridge University Press, Cambridge.

Davydenko, S. A., Strebulaev, I. A., and Zhao, X. (2012). A market-based study of the cost of default. The Review of Financial Studies, 25(10):2959-2999.

De Souza, S. R. S., Silva, T. C., Tabak, B. M., and Guerra, S. M. (2016). Evaluating systemic risk using bank default probabilities in financial networks. Journal of Economic Dynamics and Control, 66:54-75.

Degryse, H., Nguyen, G., et al. (2007). Interbank exposures: An empirical examination of contagion risk in the belgian banking system. International Journal of Central Banking, 3(2):123-171.

Eisenberg, L. and Noe, T. H. (2001). Systemic risk in financial systems. Management Science, 47(2):236-249.

Elliott, M., Golub, B., and Jackson, M. O. (2014). Financial networks and contagion. The American Economic Review, 104(10):3115-3153.

Elsinger, H., Lehar, A., and Summer, M. (2006a). Risk assessment for banking systems. Management Science, 52(9):1301-1314.

Elsinger, H., Lehar, A., and Summer, M. (2006b). Using market information for banking system risk assessment. International Journal of Central Banking, March.

Fink, K., Krüger, U., Meller, B., and Wong, L.-H. (2016). The credit quality channel: Modeling contagion in the interbank market. Journal of Financial Stability, 25:83-97.

Fischer, T. (2014). No-Arbitrage Pricing Under Systemic Risk: Accounting for CrossOwnership. Mathematical Finance, 24(1):97-124. 
Freixas, X., Parigi, B. M., and Rochet, J.-C. (2000). Systemic risk, interbank relations, and liquidity provision by the central bank. Journal of Money, Credit and Banking, $32(3): 611-638$.

Furfine, C. H. (2003). Interbank Exposures: Quantifying the risk of contagion. Journal of Money, Credit and Banking, 35(1):111-129.

Gai, P. and Kapadia, S. (2010). Contagion in financial networks. Proceedings of the Royal Society of London A, rspa20090410.

Gandy, A. and Veraart, L. A. (2016). A bayesian methodology for systemic risk assessment in financial networks. Management Science, 63(12):4428-4446.

Gauthier, C., Lehar, A., and Souissi, M. (2012). Macroprudential capital requirements and systemic risk. Journal of Financial Intermediation, 21(4):594-618.

Glasserman, P. (2005). Measuring marginal risk contributions in credit portfolios. Available from: https://ssrn.com/abstract=681227.

Glasserman, P. and Young, H. P. (2015). How likely is contagion in financial networks? Journal of Banking \& Finance, 50:383-399.

Glasserman, P. and Young, H. P. (2016). Contagion in financial networks. Journal of Economic Literature, 54(3):779-831.

Greenwood, R., Landier, A., and Thesmar, D. (2015). Vulnerable banks. Journal of Financial Economics, 115(3):471-485.

Halaj, G. (2018). Agent-based model of system-wide implications of funding risk. Available from: https://ssrn. com/abstract=3100026.

Hałaj, G. and Kok, C. (2013). Assessing interbank contagion using simulated networks. Computational Management Science, 10(2-3):157-186.

Huang, X., Zhou, H., and Zhu, H. (2012). Assessing the systemic risk of a heterogeneous portfolio of banks during the recent financial crisis. Journal of Financial Stability, 8(3):193-205.

Hüser, A.-C., Hałaj, G., Kok, C., Perales, C., and van der Kraaij, A. (2018). The systemic implications of bail-in: a multi-layered network approach. Journal of Financial Stability, 38:81-97.

Jackson, M. O. and Pernoud, A. (2019). What makes financial networks special? distorted investment incentives, regulation, and systemic risk measurement. Available from: https://ssrn.com/abstract=3311839. 
James, C. (1991). The losses realized in bank failures. The Journal of Finance, 46(4):1223-1242.

Kurth, A. and Tasche, D. (2003). Credit portfolio risk: Contributions to credit risk. Risk, 16(3):84-88.

Merton, R. C. (1974). On the pricing of corporate debt: The risk structure of interest rates. The Journal of Finance, 29(2):449-470.

Mistrulli, P. E. (2011). Assessing financial contagion in the interbank market: Maximum entropy versus observed interbank lending patterns. Journal of Banking ES Finance, 35(5):1114-1127.

Nier, E., Yang, J., Yorulmazer, T., and Alentorn, A. (2007). Network models and financial stability. Journal of Economic Dynamics and Control, 31(6):2033-2060.

Rogers, L. C. and Veraart, L. A. (2013). Failure and rescue in an interbank network. Management Science, 59(4):882-898.

Shapley, L. S. (1953). A value for n-person games. In Kuhn, H. W. and Tucker, A. W., editors, Contributions to the Theory of Games, chapter 17, pages 307-318. Princeton University Press, Princeton.

Shin, H. S. (2009). Reflections on Northern Rock: the bank run that heralded the global financial crisis. The Journal of Economic Perspectives, 23(1):101-119.

Suzuki, T. (2002). Valuing corporate debt: the effect of cross-holdings of stock and debt. Journal of the Operations Research Society of Japan, 45(2):123-144.

Tarashev, N., Borio, C., and Tsatsaronis, K. (2010). Attributing systemic risk to individual institutions. Available from: https://ssrn.com/abstract=1631761.

Upper, C. (2011). Simulation methods to assess the danger of contagion in interbank markets. Journal of Financial Stability, 7(3):111-125.

Upper, C. and Worms, A. (2004). Estimating bilateral exposures in the german interbank market: Is there a danger of contagion? European Economic Review, 48(4):827849.

Wells, S. J. (2004). Financial interlinkages in the United Kingdom's interbank market and the risk of contagion. Available from: http://www.bankofengland.co.uk/ archive/Documents/historicpubs/workingpapers/2004/wp230.pdf. 